\title{
Structure and stellar population of ringed barred galaxy NGC 2336^
}

\author{
A. S. Gusev ${ }^{1,2,3}$ and M.-G. Park ${ }^{2}$ \\ 1 Korea Astronomy Observatory, 61-1 Whaam-Dong, Yuseong-Gu, Taejon 305-348, Korea \\ 2 Department of Astronomy and Atmospheric Sciences, Kyungpook National University, Taegu 702-701, Korea \\ 3 Sternberg Astronomical Institute, Moscow University, Universitetskii Pr. 13, Moscow 119992, Russia
}

Received 9 November 2001 / Accepted 30 July 2003

\begin{abstract}
Results are presented for surface UBVRI photometry of the giant spiral galaxy NGC 2336. The data were obtained with the 1.8-m telescope of the Bohyunsan Optical Astronomy Observatory in Korea, using a CCD camera. The structure and radial light distribution of the galaxy were studied. The compositions of the stellar populations in various regions of the galaxy were estimated using two-color diagrams, and regions of star formation were identified. The properties of the 34 largest such regions were determined. The ages of these star formation regions were estimated using stellar evolutionary synthesis models. It is shown that active, constant, non-burst star formation has been recently proceeding in the disk of NGC 2336.
\end{abstract}

Key words. galaxies: spiral - galaxies: individual: NGC 2336 - galaxies: photometry - galaxies: structure galaxies: stellar content

\section{Introduction}

NGC 2336 is a giant, ringed, barred galaxy. It has highly developed spiral structure (Fig. 1). The basic parameters of NGC 2336 from the LEDA Database (see Paturel et al. 1997) are presented in Table 1 (in this paper, a Hubble constant of $H_{0}=75 \mathrm{~km} \mathrm{~s}^{-1} \mathrm{Mpc}^{-1}$ has been assumed).

This galaxy has been studied in the optical, infrared (IR) and radio wavelengths, including surface photometry in $B$ (Boroson 1981; Blackman \& van Moorsel 1984), in $V$ (Heraudeau \& Simien 1996; Baggett et al. 1998), in $R_{J}$ (Grosbol 1985) and in $J K$ (Wilke et al. 1999), as well as aperture photometry in $H$ (Aaronson et al. 1982). Optical (3400$6700 \AA$ ) spectra of NGC 2336 have been studied by Tifft (1982) and Schechter (1983). Observations in the 12-120 $\mu \mathrm{m}$ range were obtained by Wunderlich \& Klein (1991), Keel (1993) and van Driel et al. (1993). NGC 2336 has also been observed at radio wavelengths of: $2.6 \mathrm{~mm}$ (Young et al. 1995), $2.8 \mathrm{~cm}$ (Niklas et al. 1995), $6.3 \mathrm{~cm}$ (Heckman 1980; Niklas et al. 1995) and $21 \mathrm{~cm}$ (see, e.g., Aaronson et al. 1982 and van Moorsel 1983). However, to the best of our knowledge, multicolor surface photometry of NGC 2336 has not yet been published.

The galaxies NGC 2336 and IC 467 form a wide pair (van Moorsel 1983). The projected distance between NGC 2336 and IC 467 is $20^{\prime}$ (van Moorsel 1983), corresponding to a linear scale of $180 \mathrm{kpc}$ at the distance assumed in van Moorsel

\footnotetext{
Send offprint requests to: A. Gusev, e-mail: gusev@sai .msu.ru

$\star$ Tables 2 and 5 are only available in electronic form at http://www . edpsciences.org
}

Table 1. Basic parameters of NGC 2336 (from LEDA).

\begin{tabular}{ll}
\hline \hline Parameter & Value \\
\hline Type & $\mathrm{SAB}(\mathrm{r}) \mathrm{bc}$ \\
Total apparent $B$ magnitude $\left(m_{B}\right)$ & 11.12 \\
Absolute $B$ magnitude $\left(M_{B}^{0}\right)$ & -22.32 \\
Radial velocity with respect & \\
to the Local Group $\left(V_{\mathrm{LG}}\right)$ & $2417.7 \mathrm{~km} \mathrm{~s}^{-1}$ \\
Distance $(R)$ & $32.2 \mathrm{Mpc}$ \\
Apparent diameter $\left(D_{25}\right)$ & 6.31 \\
Apparent corrected diameter $\left(d_{25}\right)$ & $64.9 \mathrm{kpc}$ \\
Inclination $(i)$ & 57.25 \\
Position angle $(\mathrm{PA})$ & $178^{\circ}$ \\
\hline
\end{tabular}

$(R=30.5 \mathrm{Mpc})$. A radial velocity difference of $\Delta V_{\mathrm{r}}=$ $159 \mathrm{~km} \mathrm{~s}^{-1}$ exists between the pair (van Moorsel 1987). However, IC 467 does not influence the structure and kinematics of NGC 2336 (Schneider \& Salpeter 1992; Keel 1993).

NGC 2336 has a non-active nucleus: strong nuclear emission has not been detected in either the optical (Tifft 1982; Heckman 1980), IR (Quillen et al. 2001) or radio (Heckman 1980; Hummel et al. 1985; Niklas et al. 1995) wavelengths. The nucleus also has a non-starlike profile in the visual passband (van den Bergh 1995).

The major axis of the relatively short bar in NGC 2336 $\left(\mathrm{PA}=120^{\circ}\right)$ is approximately in line with the minor axis of the galaxy. Corrected for inclination, the angular radius of the bar is $35^{\prime \prime}-37^{\prime \prime}$ (Boroson 1981; Wilke et al. 1999). The apparent bar axis ratio is $b / a=0.6 \pm 0.1$; corrected for inclination, it is 


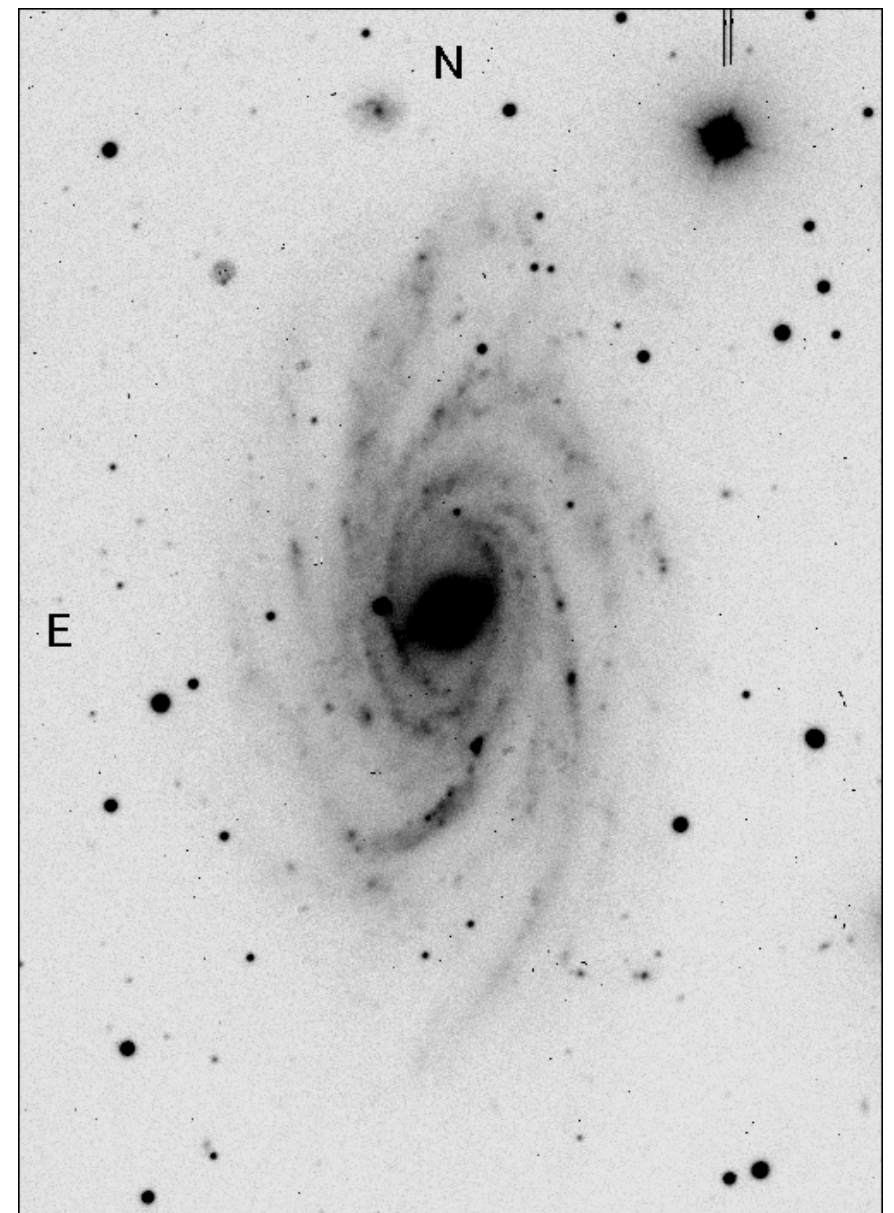

Fig. 1. 8. $0 \times$ 5.7 CCD image of NGC 2336 in the $V$ passband.

$b / a(i)=0.35 \pm 0.07$ (Martin 1995; Wilke et al. 1999). The ring radius $\left(32^{\prime \prime}-35^{\prime \prime}\right)$ is approximately equal to the bar semi-length (Boroson 1981; Buta \& de Vaucouleurs 1983). Several developed, symmetrical spiral arms spring from the ring (Boroson 1981; Biviano et al. 1991).

A bulge-disk decomposition of the radial light profiles of NGC 2336 has been carried out in the $B, V, R_{J}$ and $J$ passbands (Boroson 1981; Baggett et al. 1998; Grosbol 1985; Wilke et al. 1999). However, both the disk and bulge parameter values differ among these papers: for example, the disk scale length was estimated to be $55^{\prime \prime}$ by Baggett et al. (1998) in the $V$ passband, as well as by Grosbol (1985) in the $R_{J}$ passband, whereas Boroson (1981) found it to be $70^{\prime \prime}$ in the $B$ passband. The bulge contributes about 5-6\% of the total luminosity of the galaxy in the visible passbands (Boroson 1981; Grosbol 1985), and 21\% in the $J$ passband (Wilke et al. 1999). The fractional contribution from the bar is $14 \%$ of the total luminosity of NGC 2336 (Wilke et al. 1999).

In general, NGC 2336 is characterized as a galaxy with symmetrical structure and regular kinematics (van Moorsel 1983; Keel 1993, 1996). Apparent deviations from the symmetrical velocity field were detected only at an angular distance of 40" north of the center (van Moorsel 1983). However, the H I distribution is clearly symmetric (van Moorsel 1983). The eastern part of NGC 2336 is inclined away from us (Keel 1993).
The total mass of NGC 2336 is $4.1 \times 10^{11} M_{\odot}$ within a 4.'1 radius (van Moorsel 1983). A study of the NGC 2336-IC 467 system shows that its minimum orbital mass is $2.2 \pm 0.5$ times the sum of the individual galaxy masses (van Moorsel 1983). Wilke et al. (1999) conducted a bulge-bar-disk mass decomposition within a 1.8 radius, and estimated the total mass of NGC 2336 to be $1.60 \times 10^{11} M_{\odot}$, the bulge mass to be $1.60 \times 10^{10} M_{\odot}$, and the bar mass to be $1.51 \times 10^{10} M_{\odot}$.

NGC 2336 is a gas-rich galaxy. Its H I mass is (1.96 \pm $0.11) \times 10^{10} M_{\odot}($ Wunderlich \& Klein 1991; Young et al. 1996; Martin 1998), and that of $\mathrm{H}_{2}$ is $8.26 \times 10^{9} M_{\odot}$ (Young et al. 1996). The detected neutral hydrogen stretches out to the twice the optical radius of the galaxy (Martin 1998). Young et al. (1995) found a molecular ring with a radius of $87^{\prime \prime}$, which is 2.5 times that of the optical ring.

The galaxy has a relatively high $\mathrm{H}_{\alpha}$ luminosity of $L\left(\mathrm{H}_{\alpha}\right)=$ $3.36 \times 10^{8} L_{\odot}$, or $0.04 L_{B}$ (Young et al. 1996). In contrast, the far-infrared (FIR) luminosity of NGC $2336\left(L_{\mathrm{FIR}}=(1.1 \pm\right.$ $0.3) \times 10^{10} L_{\odot}$ or $0.2 L_{B}$ ) is lower than typical values for $\mathrm{Sbc}$ type galaxies (van Driel et al. 1993; Xu et al. 1994; Young et al. 1996). This is probably due to the presence of a cold dust component in the galaxy (Wunderlich \& Klein 1991; van Driel et al. 1993). The FIR flux ratio is only $S_{60} / S_{100}=0.24 \pm 0.03$ (van Driel et al. 1993; Young et al. 1996). The effective temperature of the dust is $T_{\text {dust }}=24 \pm 2 \mathrm{~K}$ (Wunderlich \& Klein 1991). The dust mass was estimated to be $9.7 \times 10^{6} M_{\odot}$ (Young et al. 1996).

The azimuthal distributions of the $\mathrm{H}_{2}$ gas and $\mathrm{H}_{\alpha}$ line flux are not symmetrical. A $10-20 \%$ excess of $\mathrm{H}_{2}$, and a $30 \%$ deficiency of $\mathrm{H}_{\alpha}$ flux, have been detected in the northern part of the galaxy (Rownd \& Young 1999).

Active star formation processes have been noted in NGC 2336. Hodge \& Kennicutt (1983) found 49 H II regions in this galaxy. The star formation rate (SFR) was estimated to be $9.1 M_{\odot} \mathrm{yr}^{-1}$ from FIR observations (Young et al. 1996) and $11.5 M_{\odot} \mathrm{yr}^{-1}$ from $\mathrm{H}_{\alpha}$ line observations (Rownd \& Young 1999). The corresponding star formation efficiencies (SFEs) are $3.1 \times 10^{-10} \mathrm{yr}^{-1}$ and $4.0 \times 10^{-10} \mathrm{yr}^{-1}$, respectively (converting from the SFRs, and assuming the estimated masses of $\mathrm{H} \mathrm{I}$ and $\mathrm{H}_{2}$ ). In 1987, a supernova burst was detected 1'.4 southeast of the galaxy center (Bartunov et al. 1994).

With the adopted Hubble constant of $H_{0}=$ $75 \mathrm{~km} \mathrm{~s}^{-1} \mathrm{Mpc}^{-1}$, we estimate a linear scale of $156.3 \mathrm{pc} / \mathrm{arcsec}$, at the assumed distance $(R=32.2 \mathrm{Mpc})$ to NGC 2336 .

\section{Observations and data reduction}

\subsection{Observations}

UBVRI CCD observations of NGC 2336 were made on 2001 February 22/23, using the 1.8-m telescope of the Bohyunsan Optical Astronomy Observatory (BOAO), with a CCD camera at the Ritchey-Chrétien $\mathrm{f} / 8$ focus. The camera was a SITe ARcoated $2048 \times 2048$ pixel array, with a plate scale of 0 !' $34 /$ pixel. The field of view was about $11.7 \times 11$.7. The average seeing was about 1.'9. Two exposures in each of the $B, V, R$ and $I$ filters, and three exposures in the $U$ filter, were obtained. The journal of observations is given in Table 2. 
Table 2. Journal of observations of NGC 2336 (available in electronic form).

\begin{tabular}{ccccc}
\hline \hline Filter & $\begin{array}{c}\text { Exposure } \\
\text { time }\end{array}$ & $\begin{array}{c}\text { Start UT } \\
\text { 2001 Feb. 22 }\end{array}$ & Seeing & Airmass \\
\hline
\end{tabular}

Table 3. Galactic and internal absorption for NGC 2336.

\begin{tabular}{cll}
\hline \hline $\begin{array}{c}\text { Passband, } \\
\text { color indices }\end{array}$ & $\begin{array}{l}E_{0} \\
\text { mag }\end{array}$ & $\begin{array}{l}E_{i} \\
\text { mag }\end{array}$ \\
\hline$B$ & 0.46 & 0.39 \\
$U-B$ & 0.075 & 0.063 \\
$B-V$ & 0.112 & 0.095 \\
$V-R$ & 0.055 & 0.047 \\
$V-I$ & 0.134 & 0.113 \\
\hline
\end{tabular}

The BOAO filters used with the CCD detector together formed a photometric system close to the standard JohnsonCousins UBVRI system.

\subsection{Data reduction}

The data reduction was carried out using standard techniques, with the ESO-MIDAS image processing system. The main image reduction stages were as follows:

(a) Determining and correcting for the bias and flat field;

(b) Correcting for bad pixels and dead columns (i.e. pixels containing no or spurious signals);

(c) Determining the sky background, then subtracting it from each image frame;

(d) Aligning the images;

(e) Adding those galaxy images taken with the same filter;

(f) Determining and applying the color equations;

(g) Absolute calibration, and transformation of the instrumental values to a suitable logarithmic scale (i.e. with units of $\operatorname{mag} \operatorname{arcsec}^{-2}$ ).

The translation of the instrumental ubvri magnitudes to the standard Johnson-Cousins UBVRI system was carried out using the following color equations:

$$
\begin{aligned}
& U=(0.944 \pm 0.033) u+(0.083 \pm 0.009)(u-b)+C_{u} \\
& B=(0.992 \pm 0.009) b+(0.150 \pm 0.019)(b-v)+C_{b} \\
& V=(1.012 \pm 0.002) v-(0.148 \pm 0.005)(b-v)+C_{v} \\
& R=(1.015 \pm 0.001) r+(0.062 \pm 0.003)(v-r)+C_{r} \\
& I=(1.020 \pm 0.015) i+(0.049 \pm 0.029)(v-i)+C_{i} .
\end{aligned}
$$

These were constructed using observations, made on the same night, of the field of the star PG $0918+029$, for which a photometric calibration has been determined by Landolt (1992). The equations are true for the airmass value 1.4. In addition, the published UBVRI aperture photometry for NGC 2336 (Prugniel \& Heraudeau 1998) was used for the absolute calibration of the galaxy. The zero-point errors were found to be: $\Delta U=0.022 \mathrm{mag}, \Delta B=0.012 \mathrm{mag}, \Delta V=0.040 \mathrm{mag}$, $\Delta R=0.047 \mathrm{mag}$ and $\Delta I=0.052 \mathrm{mag}$.

We studied the structure and photometric parameters of NGC 2336 on various scales $>2$ " (the observational seeing, see Table 2). The images were smoothed using different sized boxes, for display in the figures: smoothing for the photometric profiles of NGC 2336 is $7 \times 7$ pixels $\left(22^{\prime \prime} 3 \times 2\right.$ '. 3$)$ in the $U$ and $B$ passbands (Figs. $2 \mathrm{a}, \mathrm{c})$, and $3 \times 3$ pixels $\left(1{ }^{\prime \prime} 0 \times 1{ }^{\prime \prime} 0\right)$ in the $V, R$ and $I$ passbands (Figs. $2 \mathrm{a}-\mathrm{d}$ ). Smoothing for the isophotal maps is $7 \times 7$ pixels $\left(2^{\prime \prime} .3 \times 2^{\prime \prime} .3\right)$ in both $B$ and $I$ passbands (Figs. 3a, b). The smoothing box for the color maps and color profiles of the galaxy is $15 \times 15$ pixels $\left(5^{\prime \prime} .0 \times 5^{\prime \prime}\right.$. 0$)$ for all color indices (Figs. 5a-d, 6a-d). The photometric accuracies of the smoothed images are: $\Delta U=0.14 \mathrm{mag}, \Delta B=0.07 \mathrm{mag}$, $\Delta V=0.05 \mathrm{mag}, \Delta R=0.04 \mathrm{mag}, \Delta I=0.04 \mathrm{mag}, \Delta(U-B)=$ $0.11 \mathrm{mag}, \Delta(B-V)=0.06 \mathrm{mag}, \Delta(V-R)=0.04 \mathrm{mag}$ and $\Delta(R-I)=0.04 \mathrm{mag}$. The values show the accuracies for the profiles and maps in Figs. 2a-d, 3a, 3b, 5a-d, 6a-d only. They are not valid for any other data.

In this paper, we corrected all data for Galactic absorption, following the standard procedure given in the RC3 Catalogue (de Vaucouleurs et al. 1991). The data in Sects. 3.4 and 3.5 were additionally corrected for dust absorption due to the inclination of NGC 2336; these inclination-corrected values are indicated by an " $i$ " superscript: " $C I_{0}^{i}$ ". The corrections in the standard Johnson-Cousins system associated with Galactic absorption $\left(E_{0}\right)$, and with the transformation of quantities to their "faceon" values $\left(E_{i}\right)$, were calculated using information from the LEDA Database; they are presented in Table 3.

\section{Results}

\subsection{Photometric profiles and morphology of the galaxy}

A small starlike nucleus of $0.8 \mathrm{kpc}\left(5^{\prime \prime}\right)$ diameter appears in the photometric profiles presented in Figs. 2a-d. The surface brightness of the nucleus reaches a maximum of $18.3 \mathrm{mag} \mathrm{arcsec}^{-2}$ in $B$. In contrast, a large bulge is observed, with a radius of $2.6 \mathrm{kpc}\left(17^{\prime \prime}\right)$.

The major axis of the short bar of the galaxy lies roughly in the same direction as the minor axis of NGC 2336 (Figs. 3a, b). The apparent semi-length of the bar is $24^{\prime \prime}$ (Figs. 2c, d), corresponding to a deprojected distance of $5.9 \mathrm{kpc}(37.5)$. The western part of the bar appears to be smaller than the eastern part, in the longer wavelength passbands (see Figs. 2d, 3b). In the bluer passbands, this bar asymmetry is less pronounced (Figs. 2c, 3a). A probable reason for this is the presence of more active star formation in the eastern arm of the bar (see Sect. 3.4 for more details). The bar of NGC 2336 has an exponentiallike intensity profile, with a scale length for the eastern arm of $\alpha^{-1}=2.8 \pm 0.3 \mathrm{kpc}\left(18^{\prime \prime} \pm 2^{\prime \prime}\right)$ in the $I$ passband. The average surface brightness of the bar, $21.4 \pm 0.2 \mathrm{mag} \mathrm{arcsec}^{-2}$ in $B$ and $20.6 \pm 0.2 \mathrm{mag} \mathrm{arcsec}^{-2}$ in $V$ (see Fig. 2c), is typical of bars.

In our images of NGC 2336, the ring is not an easily distinguished structural unit (Figs. 1, 2a, 2b, 3a). We estimate its radius to be $5.3 \pm 0.5 \mathrm{kpc}\left(34^{\prime \prime} 1 \pm 2\right.$ '. 3$)$. The outer ring radius is about equal to the bar semi-length. The surface brightness of the ring reaches $21.8 \pm 0.2 \mathrm{mag} \mathrm{arcsec}^{-2}$ in $B$, which is only $0.2-0.3 \mathrm{mag} \mathrm{arcsec}^{-2}$ higher than the surface brightness of the background to the ring (Fig. 2a). This difference is even smaller in the redder passbands (see Figs. 2a, b). 

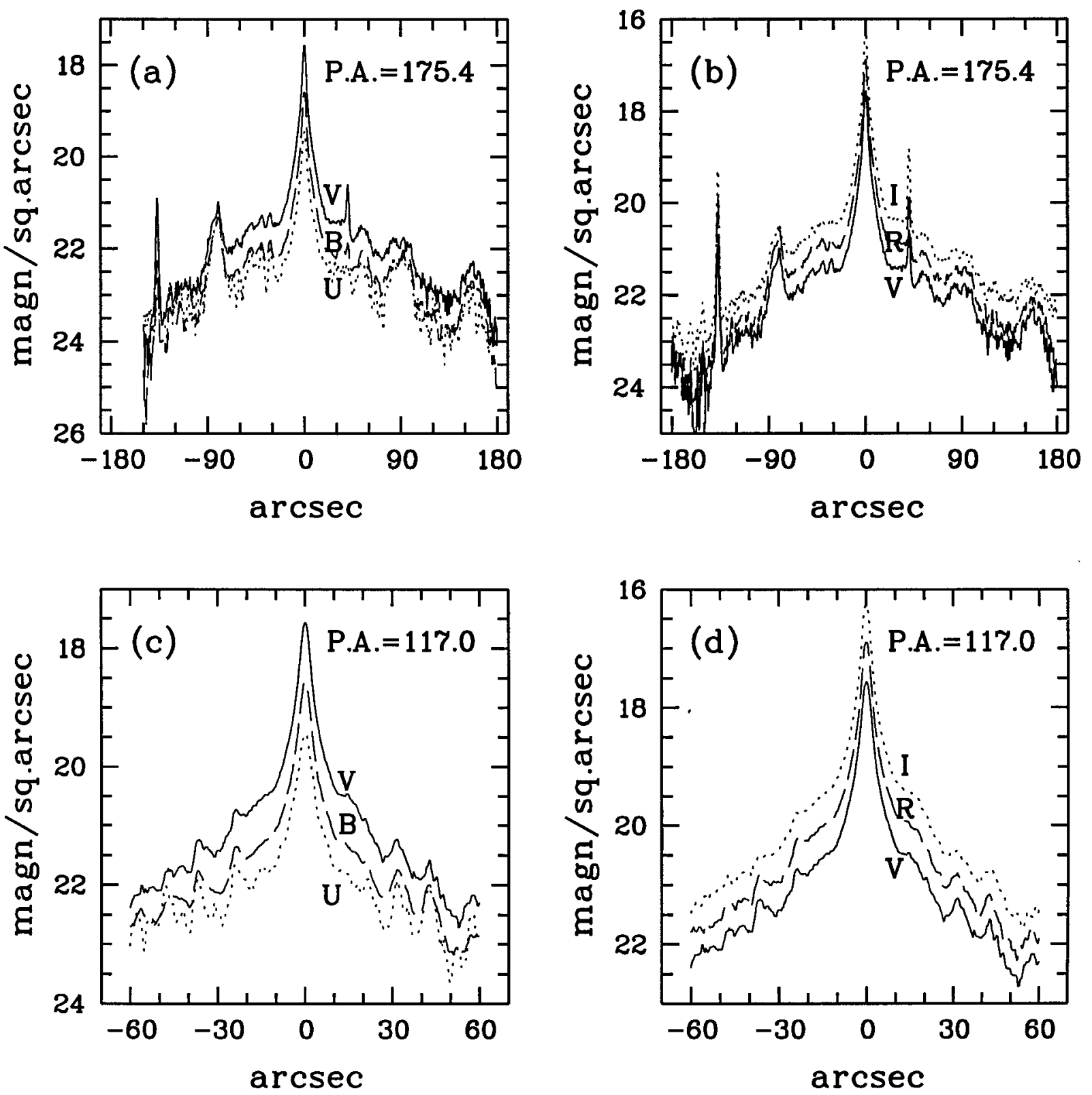

Fig. 2. NGC 2336 photometric profiles (in mag $\operatorname{arcsec}^{-2}$ ) along the major axis of the galaxy (PA = 175:4) (a), b)), and along the major axis of the $\left.\left.\operatorname{bar}\left(\mathrm{PA}=117^{\circ} .0\right)(\mathbf{c}), \mathbf{d}\right)\right)$, in the $U$ (dotted line), $B$ (dashed line), $V$ (solid line) $\left.(\mathbf{a}), \mathbf{c}\right)$ ), and $V$ (solid line), $R$ (dashed line), and $I($ dotted line) (b), d)) passbands. Negative angular distances correspond to the southern (a), b)) and the eastern (c), d)) parts of NGC 2336. These profiles cut across the galactic components at the following locations: (a), b)) nucleus $\left(r=0^{\prime \prime} \pm 2^{\prime \prime}\right)$, bulge $\left(r= \pm 4^{\prime \prime} \pm 16^{\prime \prime}\right)$, ring $\left(r= \pm 32^{\prime \prime} \pm 36^{\prime \prime}\right)$, external disk $\left(r= \pm 105^{\prime \prime} \pm 180^{\prime \prime}\right)$, spiral arms $\left(r=-80^{\prime \prime},-45^{\prime \prime},+55^{\prime \prime},+95^{\prime \prime},+160^{\prime \prime}\right)$; (c), d) ) nucleus $\left(r=0^{\prime \prime} \pm 2^{\prime \prime}\right)$, bulge $\left(r= \pm 4^{\prime \prime} \pm 10^{\prime \prime}\right)$, bar $\left(r= \pm 13^{\prime \prime} \pm 22^{\prime \prime}\right)$, ring $\left(r= \pm 23^{\prime \prime}\right)$, spiral arms $\left(r=-57^{\prime \prime},-47^{\prime \prime},-42^{\prime \prime},-36^{\prime \prime},+33^{\prime \prime},+42^{\prime \prime},+57^{\prime \prime}\right)$. The two sharp-peaked maxima in (a), b)) at radii of $r=-135^{\prime \prime}$ and $r=40^{\prime \prime}$ are field stars. The coordinate accuracy is $2^{\prime \prime}$.

Several field stars are projected onto the galactic disk. One bright star is $28^{\prime \prime}$ east of the center of NGC 2336 (Figs. 1, $3 \mathrm{a}, 3 \mathrm{~b})$. Two field stars are projected onto the major axis of the galaxy (at radii of $r=-135^{\prime \prime}$ and $40^{\prime \prime}$ in Figs. 2a, b). These field stars were subtracted to allow further investigations, such as quantifying the azimuthal and radial light distributions.

NGC 2336 has numerous, long, developed and twisted spiral arms (Figs. 1, 3a, 3b). Detailed explorations of its spiral structure are difficult to perform, due to the large inclination of this galaxy. We found six distinct spiral arms in NGC 2336: four arms in the northern and western regions, one bright arm in the southern part of the galaxy, and one faint arm in the eastern part. As a result, some asymmetry is expected to appear in the photometric profiles along the major axis of the galaxy (see Figs. 2a, b). The surface brightness of the spiral arms decreases from $21.8 \pm 0.2 B$ mag arcsec ${ }^{-2}$ in the inner part of the disk, to $23.2 \pm 0.2 B$ mag arcsec ${ }^{-2}$ in the outer part. The numerous, small "hills" in Figs. 2a-d result from the spiral arms cutting across the chosen photometric profile axes.

In spite of the apparent difference in surface brightness between the western and eastern parts of NGC 2336 (Fig. 1), 

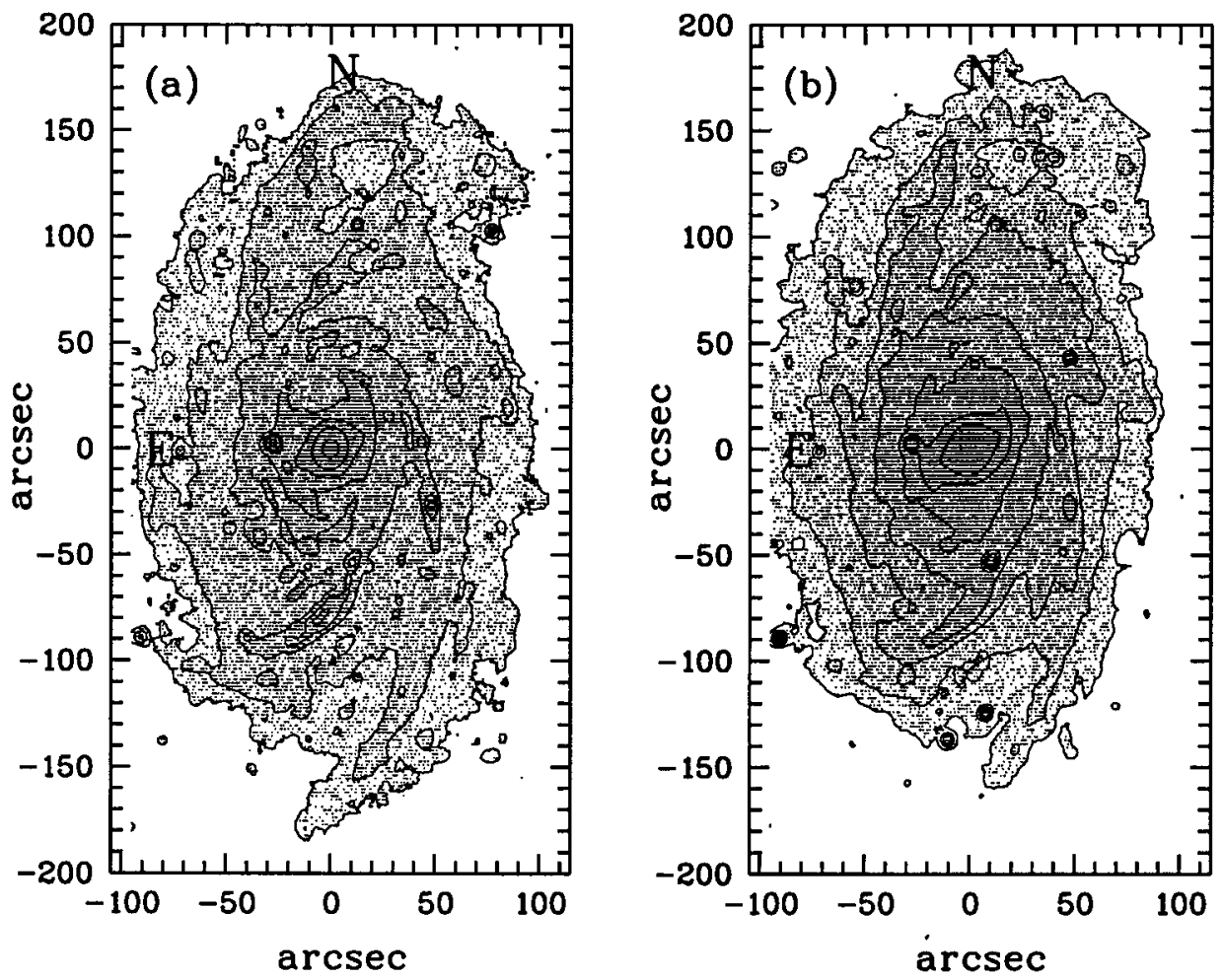

Fig. 3. Isophotal map of NGC 2336 in the a) $B$ and b) $I$ passbands. The isophotal contour levels a) 20.0, 21.0, 21.5, 22.0, 22.5, 23.5, $24.5 B$ mag $\operatorname{arcsec}^{-2}$, and b) 19.5, 20.0, 20.5, 21.0, 21.5, 22.0, $22.5 I \mathrm{mag} \operatorname{arcsec}^{-2}$, are shown.

the western part is actually less than $0.15 B$ mag $\operatorname{arcsec}^{-2}$ brighter than the eastern part (see Figs. 3a, b).

Many bright star formation regions are observed in the spiral arms of NGC 2336, and the surface brightness in these regions reaches a maximum of $20.2 \mathrm{~B} \mathrm{mag} \operatorname{arcsec}^{-2}$. The largest star formation complex is located in the southern spiral arm, at a distance of $12 \mathrm{kpc}\left(80^{\prime \prime}\right)$ south of the center of NGC 2336 (Figs. 2a, 2b, 3a). Its size is $2.4 \times 1.0 \mathrm{kpc}\left(15^{\prime \prime} \cdot 6 \times 66^{\prime \prime} 3\right)$.

The estimated position angles and ellipticities $(e \equiv 1-b / a)$ of the smoothed isophotes of NGC 2336, as a function of angular distance from the galaxy center, are presented in Figs. 4a, b. These results are in agreement with Heraudeau \& Simien (1996).

We used a smoothing box of $11 \times 11$ pixels $\left(3{ }^{\prime \prime} 7 \times 3\right.$ '.7) for studying the position angles and ellipticities in the images. The accuracy of the ellipticity functions constructed from the smoothed isophotal images is 0.04 mag in the $U$ and $R$ passbands, and $0.03 \mathrm{mag}$ in the $B, V$ and $I$ passbands.

The bulge of NGC 2336 is not circularly symmetric. Its ellipticity is $0.20 \pm 0.02$ (Fig. $4 \mathrm{~b}$ ), and its position angle is $172^{\circ}$ $\pm 2^{\circ}$, which is close to that of the galaxy as a whole (Fig. 4a).

Among the observed passbands, the isophotal position angles and ellipticities differ most notably in the angular distance range $r=20^{\prime \prime}-40^{\prime \prime}$ from the galaxy center. The relatively young stellar population of the bar and ring occupy this region. The wavelength dependence of position angle and ellipticity is a result of a change in stellar population content along the bar of NGC 2336 (see Sect. 3.4). We estimated the bar position angle to be $117.0 \pm 0.3$ in the $V, R$ and $I$ passbands, and its apparent ellipticity to be $0.33 \pm 0.06$ in the $U, B, V$ and
$R$ passbands. This result for the bar ellipticity is more precise than those of Martin (1995) and Wilke et al. (1999).

The isophotal position angles are approximately constant $\left(180^{\circ} \pm 10^{\circ}\right)$ at radial distances $\left(r>40^{\prime \prime}\right)$ where the disk dominates the light from the galaxy. A local "maximum" (PA = $189^{\circ} \pm 2^{\circ}$ ) appears at an angular distance of $55^{\prime \prime}$ from the galaxy center (Fig. 4a). The ellipticity increases from $0.32 \pm 0.02$ at $r=40^{\prime \prime}$ to $0.43 \pm 0.03$ at $r>120^{\prime \prime}$ (Fig. 4b). The ellipticity values determined from the $U$ and $B$ images are smaller than those determined from the redder passbands, in the angular range $r=60^{\prime \prime}-120^{\prime \prime}$. This region undergoes active star formation in numerous star formation complexes (see Sects. 3.4, 3.5); the chance distribution of these star formation regions distorts the ellipticity values determined from the bluer passbands.

We estimated the position angle and ellipticity for the galaxy as a whole, in the $V, R$ and $I$ passbands, as PA = $175^{\circ} .4 \pm 1^{\circ} .0$ and $e=0.42 \pm 0.02$. This ellipticity corresponds to a galactic inclination of $i=54^{\circ} .8 \pm 1.4$.

\subsection{Color distribution}

The data presented in this section, and in the next, have been corrected just for Galactic absorption.

Only regions with signal-to-noise ratio $(S N R)$ greater than 5 were studied in this section. Thus, we did not consider the $U-B$ data for the disk of NGC 2336 (see Fig. 5a).

The total color indices of NGC 2336 are typical of intermediate-type spiral galaxies: $U-B=-0.01 \mathrm{mag}, B-V=$ $0.51 \mathrm{mag}$, and $V-I=0.99 \mathrm{mag}$ (these values are from the LEDA Database). 
Table 4. Radial photometric parameters for the disk and bulge of NGC 2336.

\begin{tabular}{|c|c|c|c|c|}
\hline \multirow[b]{2}{*}{ Passband } & \multicolumn{2}{|c|}{ Disk $\left(r=68^{\prime \prime}-166^{\prime \prime}\right)$} & \multicolumn{2}{|c|}{ Bulge $\left(r=4^{\prime \prime}-16^{\prime \prime}\right)$} \\
\hline & $\begin{array}{c}\text { Scale } \\
\text { length } \\
r_{0}, \mathrm{kpc}(\operatorname{arcsec}) \\
\end{array}$ & $\begin{array}{c}\text { Central surface } \\
\text { brightness } \\
\mu_{0},{\text { mag } \operatorname{arcsec}^{-2}}\end{array}$ & $\begin{array}{c}\text { Effective } \\
\text { radius } \\
r_{\mathrm{e}}, \mathrm{kpc}(\operatorname{arcsec}) \\
\end{array}$ & $\begin{array}{c}\text { Effective surface } \\
\text { brightness } \\
\mu_{\mathrm{e}},{\text { mag } \operatorname{arcsec}^{-2}}^{-2}\end{array}$ \\
\hline$B$ & $11.55 \pm 0.30(73.92 \pm 1.91)$ & $21.33 \pm 0.06$ & $1.80 \pm 0.26(11.53 \pm 1.67)$ & $21.46 \pm 0.13$ \\
\hline$V$ & $10.58 \pm 0.17(67.71 \pm 1.07)$ & $20.73 \pm 0.04$ & $2.02 \pm 0.28(12.96 \pm 1.82)$ & $20.80 \pm 0.12$ \\
\hline$R$ & $9.66 \pm 0.15(61.81 \pm 0.93)$ & $20.19 \pm 0.04$ & $1.85 \pm 0.20(11.84 \pm 1.28)$ & $20.07 \pm 0.10$ \\
\hline$I$ & $11.04 \pm 0.12(70.61 \pm 0.79)$ & $19.95 \pm 0.03$ & $1.82 \pm 0.17(11.63 \pm 1.07)$ & $19.43 \pm 0.08$ \\
\hline
\end{tabular}
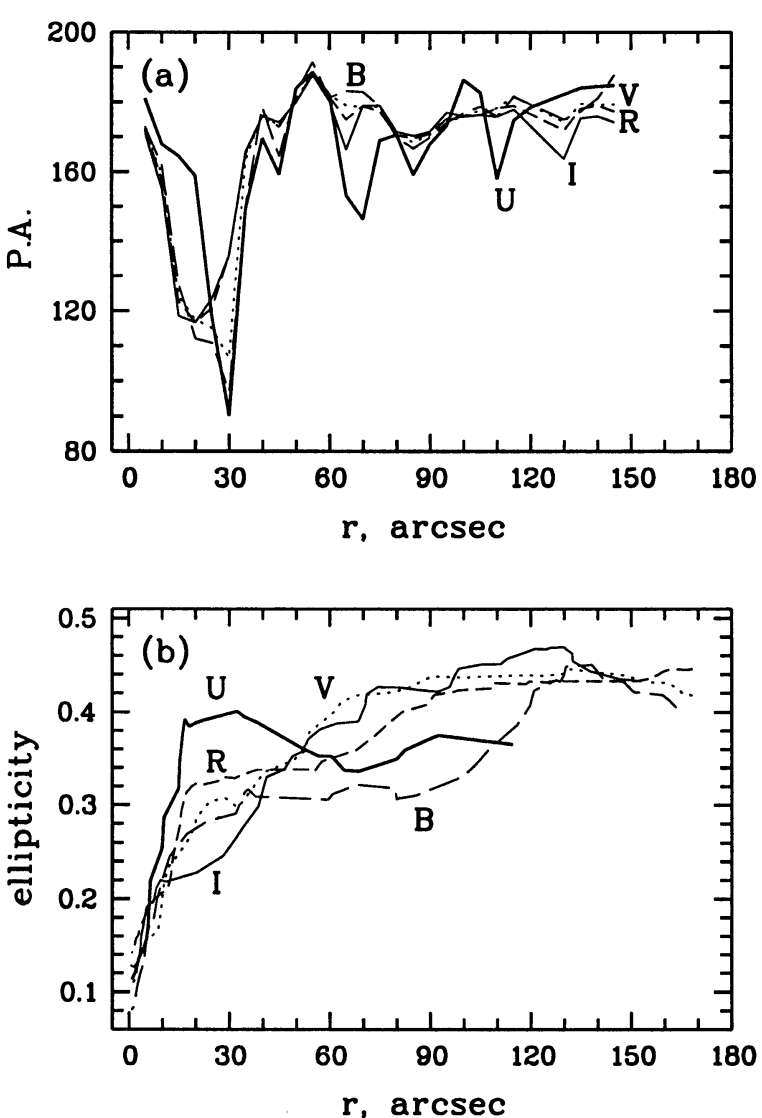

Fig. 4. The dependence of a) position angle and b) ellipticity ( $e \equiv$ $1-b / a)$ of the smoothed isophotes of NGC 2336, on the angular distance $r$ from the galaxy center, in the $U$ (thick solid line), $B$ (long dashed line), $V$ (dotted line), $R$ (short dashed line) and $I$ (thin solid line) passbands.

The nucleus and bulge of NGC 2336 are the reddest parts of the galaxy (Figs. 5a-d, 6a-d). The color indices of the bulge are: $U-B=0.5 \pm 0.1 \mathrm{mag}, B-V=0.7 \pm 0.1 \mathrm{mag}, V-R=$ $0.54 \pm 0.02 \mathrm{mag}$, and $R-I=0.55 \pm 0.01 \mathrm{mag}$. The color indices at the galaxy center reach as high as: $U-B=0.83 \mathrm{mag}$, $B-V=0.86 \mathrm{mag}, V-R=0.58 \mathrm{mag}$, and $R-I=0.63 \mathrm{mag}$. These values indicate an old stellar population in the central regions.

The ring of NGC 2336 has a relatively blue color: $U-B=$ $0.30 \pm 0.05 \mathrm{mag}, B-V=0.5 \pm 0.1 \mathrm{mag}, V-R=0.46 \pm 0.03 \mathrm{mag}$, and $R-I=0.43 \pm 0.03 \mathrm{mag}$. The color along the bar becomes progressively bluer on going from the bulge to the ring.
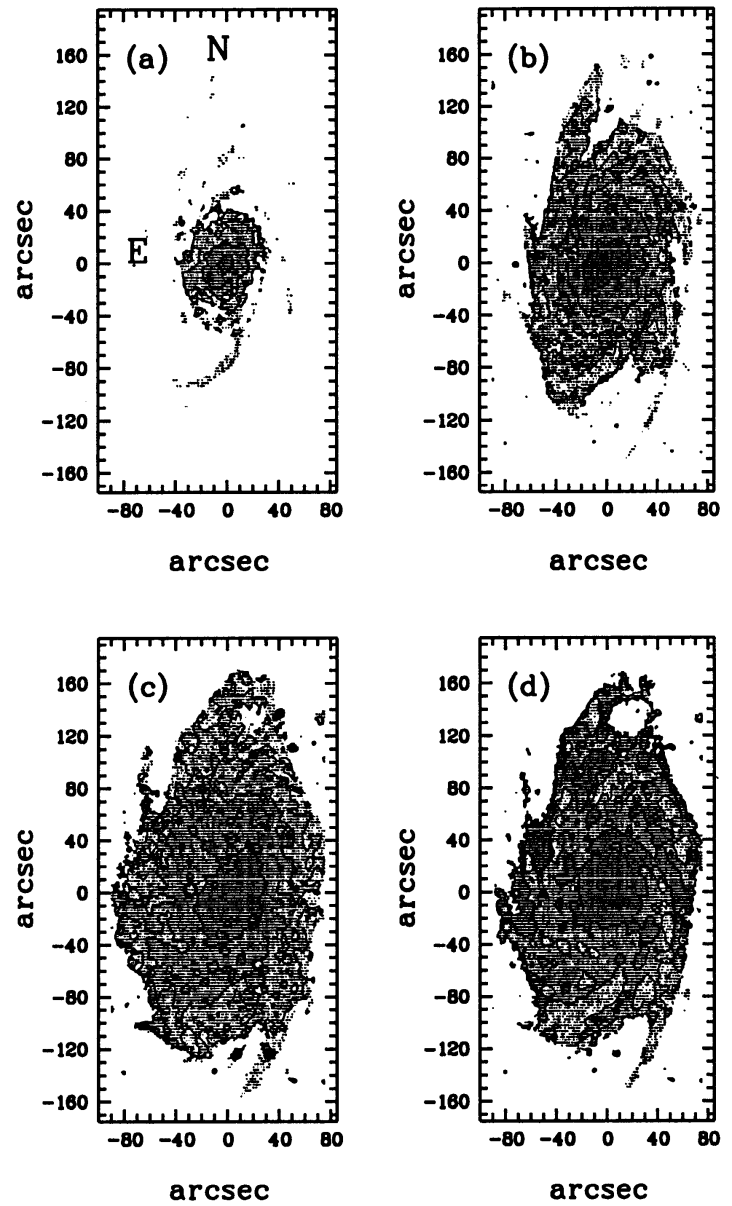

Fig. 5. NGC 2336 color index maps in a) $U-B$, b) $B-V$, c) $V-R$, and d) $R-I$. Contours of constant color index are shown, with levels set at: a) $U-B=0.2,0.4,0.6 \mathrm{mag}$, b) $B-V=0.45,0.6,0.75 \mathrm{mag}$, c) $V-R=0.35,0.42,0.5 \mathrm{mag}$, and d) $R-I=0.4,0.5 \mathrm{mag}$. Redder regions are shaded darker than bluer ones. Regions with $S N R<5$ are indicated by the white areas.

In general, the disk of NGC 2336 becomes bluer towards the periphery (Figs. $6 \mathrm{a}-\mathrm{d}$ ); the color indices decrease from 0.5 to 0.4 mag in $V-R$. However, $B-V$ and $R-I$ do not vary significantly with distance from the galaxy center (Figs. 6a, b).

The spiral arms of NGC 2336 are the bluest regions in the galaxy; this is clearly seen in Figs. 6b, d. The color indices in the spiral arm regions are: $U-B=-0.14 \pm 0.06 \mathrm{mag}$, $B-V=0.33 \pm 0.04 \mathrm{mag}, V-R=0.35 \pm 0.04 \mathrm{mag}$, and 

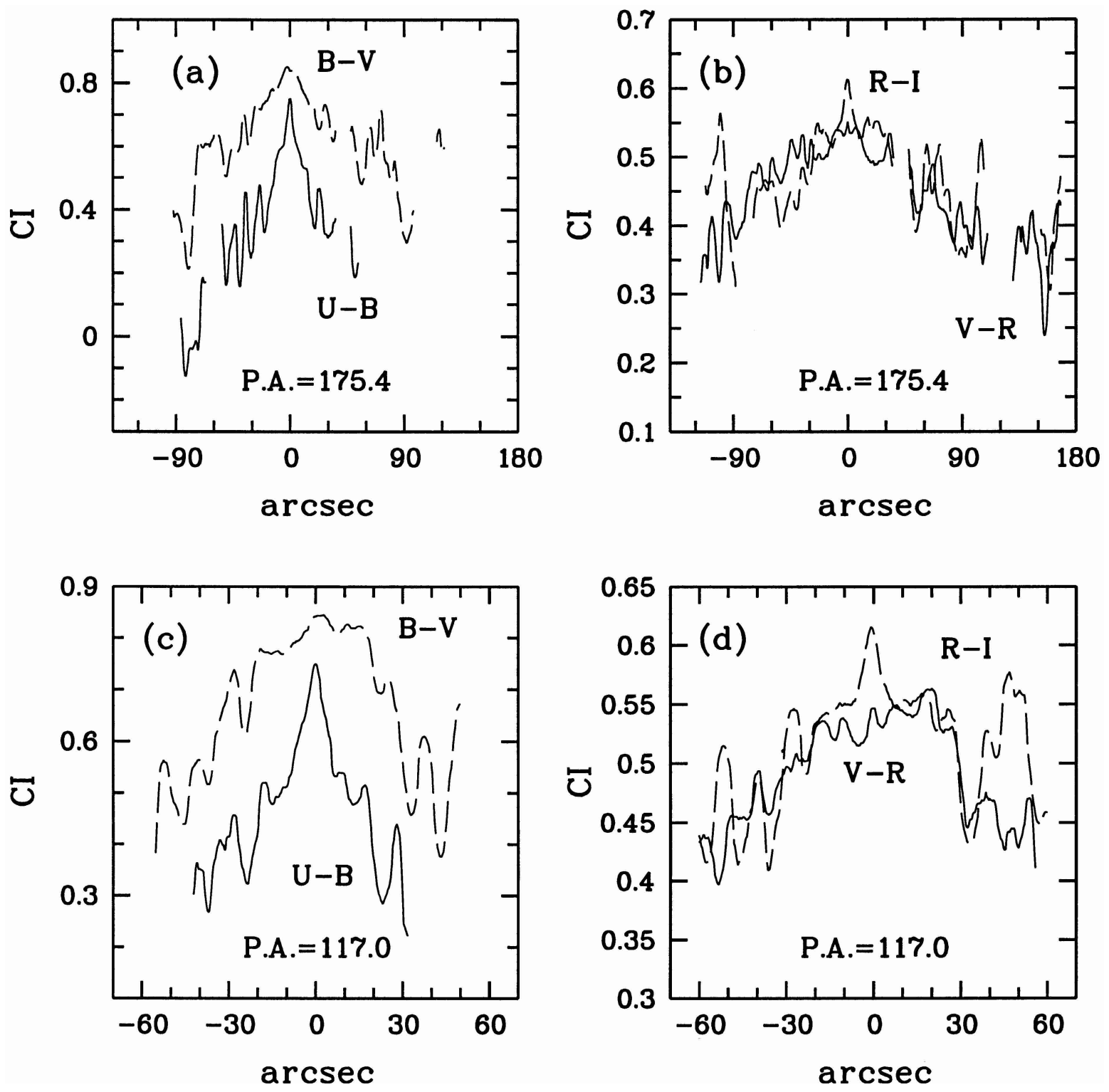

Fig. 6. NGC 2336 color profiles along the major axis of the galaxy $\left(\mathrm{PA}=175^{\circ} .4\right)(\mathbf{a})$, b) $)$, and along the major axis of the bar $\left(\mathrm{PA}=117^{\circ} .0\right)$ (c), d)), in the (a), c)) $U-B$ (solid line), $B-V$ (dashed line), $V-R$ (solid line), and $R-I$ (dashed line) (b), d)) color indices. Only profiles for regions having $S N R \geq 5$ are shown. Negative angular distances correspond to the southern (a), b)) and eastern parts (c), d)) of NGC 2336. These profiles cut across the galactic components at the following locations: (a), b)) nucleus $\left(r=0^{\prime \prime} \pm 2^{\prime \prime}\right)$, bulge $\left(r= \pm 4^{\prime \prime} \pm 16^{\prime \prime}\right)$, ring $\left(r= \pm 32^{\prime \prime} \pm 36^{\prime \prime}\right)$, external disk $\left(r= \pm 105^{\prime \prime} \pm 180^{\prime \prime}\right)$, spiral arms $\left(r=-80^{\prime \prime},-45^{\prime \prime},+55^{\prime \prime},+95^{\prime \prime},+160^{\prime \prime}\right)$; $(\mathbf{c})$, d $\left.)\right)$ nucleus $\left(r=00^{\prime \prime} \pm 2^{\prime \prime}\right)$, bulge $\left(r= \pm 4^{\prime \prime} \pm 10^{\prime \prime}\right)$, bar $\left(r= \pm 13^{\prime \prime} \pm 22^{\prime \prime}\right)$, ring $\left(r= \pm 23^{\prime \prime}\right)$, spiral arms $\left(r=-57^{\prime \prime},-47^{\prime \prime},-42^{\prime \prime},-36^{\prime \prime},+33^{\prime \prime},+42^{\prime \prime},+57^{\prime \prime}\right)$. The coordinate accuracy is $2^{\prime \prime}$.

$R-I=0.35 \pm 0.08 \mathrm{mag}$. The star formation regions have still bluer colors (see Sect. 3.5).

Strong variations in the $R-I$ color index are observed between the arm and interarm regions. $R-I$ can be as high as $0.54 \pm 0.03 \mathrm{mag}$ in the interarm regions (Figs. 6b, d). On the contrary, the $V-R$ color index is approximately the same for both arm and interarm regions (Fig. 6d); this is a typical result for spiral galaxies. There are two competing effects involved here: firstly, both the $V$ and $R$ passbands are sensitive to the presence of a young stellar population in the spiral arms, whereas the $I$ passband has relatively little such sensitivity. Secondly, there is wavelength-dependent dust absorption, which "reddens" the radiation more effectively for the shorter wavelengths; so the intrinsic color contrast variations between blue and red stellar populations will tend to be "washed out" or "smoothed" by the reddening from the intervening dust.

Regions located along the major axis of NGC 2336, at angular distances $r>90^{\prime \prime}$ to the south, and for $r=100^{\prime \prime}-150^{\prime \prime}$ to the north from the galaxy center, are among the dimmest parts of the galaxy, and so are difficult to investigate with any confidence (see Figs. 1, 2a, 2b).

The eastern (furthermost) part of NGC 2336 is redder than the western part (Figs. 5c, 6c, 6d). The $B-V$ and $V-R$ color indices of the western part are smaller by $0.1 \mathrm{mag}$ and $0.03 \mathrm{mag}$, respectively, than those of the eastern part, at apparent angular distances of $40^{\prime \prime}-70^{\prime \prime}$ from the center (i.e. $0.45 \mathrm{mag}(\mathrm{W}) \mathrm{vs}$. 

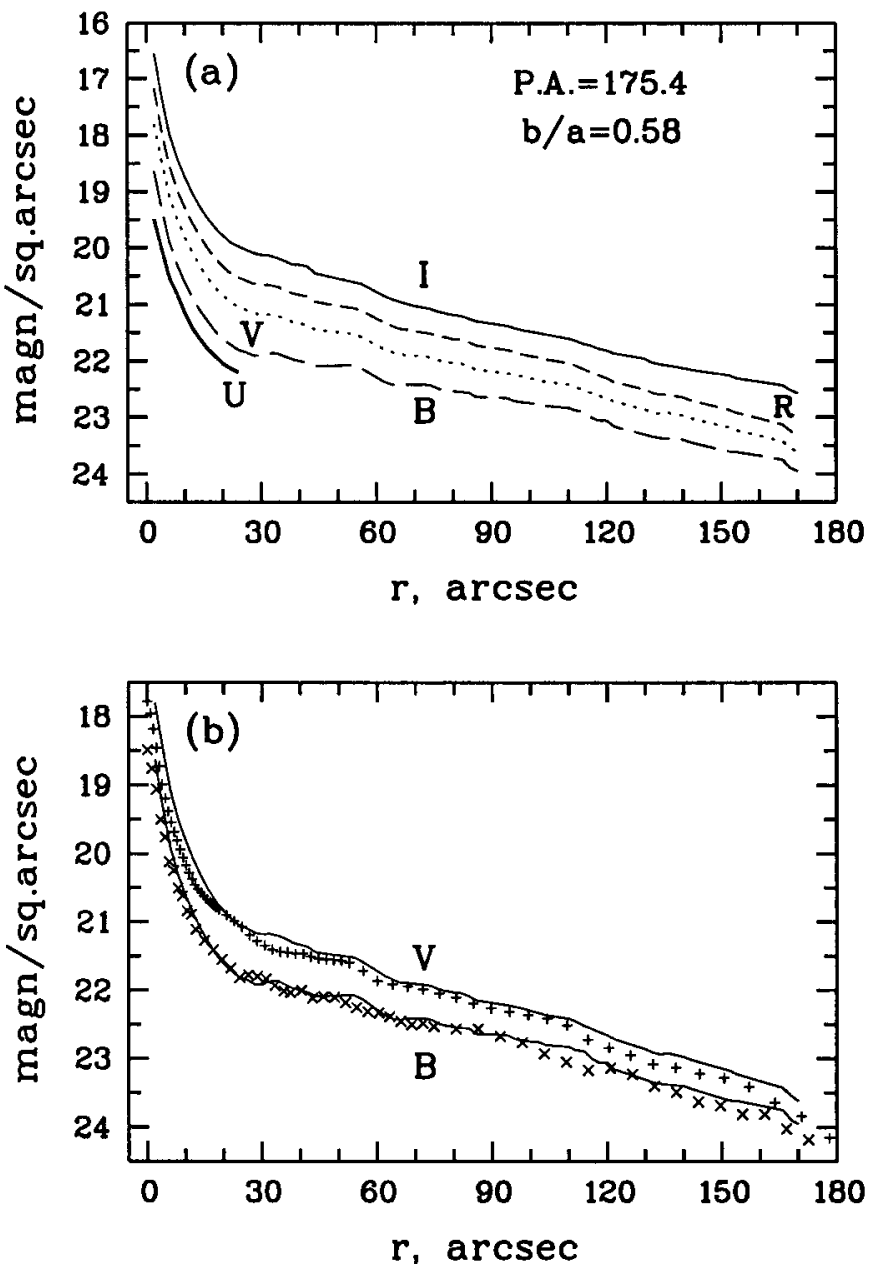

Fig. 7. a) The radial light distribution dependence on the angular distance $r$ from the center of NGC 2336, in the $U$ (thick solid line), $B$ (long dashed line), $V$ (dotted line), $R$ (short dashed line) and $I$ (thin solid line) passbands. b) Comparison of our data (solid lines) with results from Boroson (1981) for the $B$ passband (diagonal crosses), and with those from Heraudeau \& Simien (1996) for the $V$ passband (vertical crosses).

$0.55 \mathrm{mag}(\mathrm{E})$ for $B-V$, and $0.42 \mathrm{mag}(\mathrm{W}) \mathrm{vs} .0 .45 \mathrm{mag}(\mathrm{E})$ for $V-R$, respectively).

Color asymmetry is observed along both the major and the minor axes of NGC 2336 (Figs. 5b-d, 6a-d). This results from the structural asymmetry of the galaxy. Numerous spiral arms, without large star formation regions, are found in the northern part of NGC 2336, and one spiral arm with a large star formation complex exists in the southern part. The photometric profiles along the minor axis of the galaxy are crossed by the spiral arms at different distances to the east and west of the galaxy center (compare the profiles at distances of $r=20^{\prime \prime}-$ $60^{\prime \prime}$ in both directions from the center, in Figs. 2c, 2d, 6c, 6d). Moreover, the dust reddening tends to "smooth" the profile in the eastern (furthermost) part of NGC 2336.

\subsection{Radial light distribution}

The radial light distributions of NGC 2336, evaluated in steps of $2^{\prime \prime}$, are presented in Fig. 7a, assuming the PA and $b / a$ obtained for the galaxy as a whole (in Sect. 3.1). We compared our data with the results from Boroson (1981) for the $B$ passband, and with those of Heraudeau \& Simien (1996) for the $V$ passband (Fig. 7b). The relatively small discrepancies between ours and their previous results are due to the different values of $\mathrm{PA}$ and $b / a$ used.

The disk and bulge parameters, derived from the decomposition of the radial light distribution profiles in the $B, V, R$ and $I$ passbands, are presented in Table 4. An exponential model for the disk:

$I^{d}=I_{0}^{d} \exp \left(-r / r_{0}\right)$,

and a de Vaucouleurs model for the bulge:

$I^{b}=I_{0}^{b} \exp \left(-\left(r / r_{0}\right)^{1 / 4}\right)$,

were assumed. The standard King models for the bulge were found to be incompatible with our data.

The disk component of NGC 2336 is by far the dominant one for angular distances $r>40^{\prime \prime}$. However, we can visually distinguish three disk regions in Fig. 7a: $r=40^{\prime \prime}-60^{\prime \prime}$, $r=60^{\prime \prime}-110^{\prime \prime}$, and $r=110^{\prime \prime}-170^{\prime \prime}$. The slightly irregular radial light distributions in the innermost region $\left(r=40^{\prime \prime}-60^{\prime \prime}\right)$ arise from the chance distribution of bright spiral arms in the disk of NGC 2336. Similar irregularities in the middle region $\left(r=60^{\prime \prime}-110^{\prime \prime}\right)$ are due to the influence of the largest star formation complex, which resides in the southern galactic spiral arm (Fig. 3a).

In spite of these small deviations from the standard profile, the exponential disk parameters were derived from the $I$ light distribution for the distance range $r=68^{\prime \prime}-166^{\prime \prime}$; the light of the southern star formation complex is not so apparent in the I passband, and nor is that from the other regions with young stellar populations. The $I$ light distribution is expected to follow the radial mass distribution, and so it has a clear physical interpretation. For completeness, the disk parameters derived from the $B, V$ and $R$ light profiles are also presented in Table 4.

The bulge component in NGC 2336 is dominant over angular distances of $r=4^{\prime \prime}-16^{\prime \prime}$ from the galaxy center. The derived values of the effective bulge radius, in the different passbands, agree to within the estimated photometric errors (see Table 4).

The NGC 2336 bulge-disk luminosity ratio is $L_{\text {bulge }} / L_{\text {disk }}=$ $0.16 \pm 0.04$ in the $I$ passband. This value agrees with those given in Boroson (1981), Grosbol (1985) and Wilke et al. (1999).

\subsection{Two-color diagrams}

The data in this section have been additionally corrected for the internal dust absorption due to the inclination of NGC 2336; so the color indices in this section are bluer than those in Sect. 3.2, after applying both of the corrections listed in Table 3 .

The intensities of the studied regions have not been corrected for the light from the surrounding background areas. The integrated color indices of the galactic components were obtained using the radial light distribution data (Fig. 7a).

In Figs. 8a-8c, the numbers denote the following regions of the galaxy: 1 is the integrated color of the galaxy as a whole, 

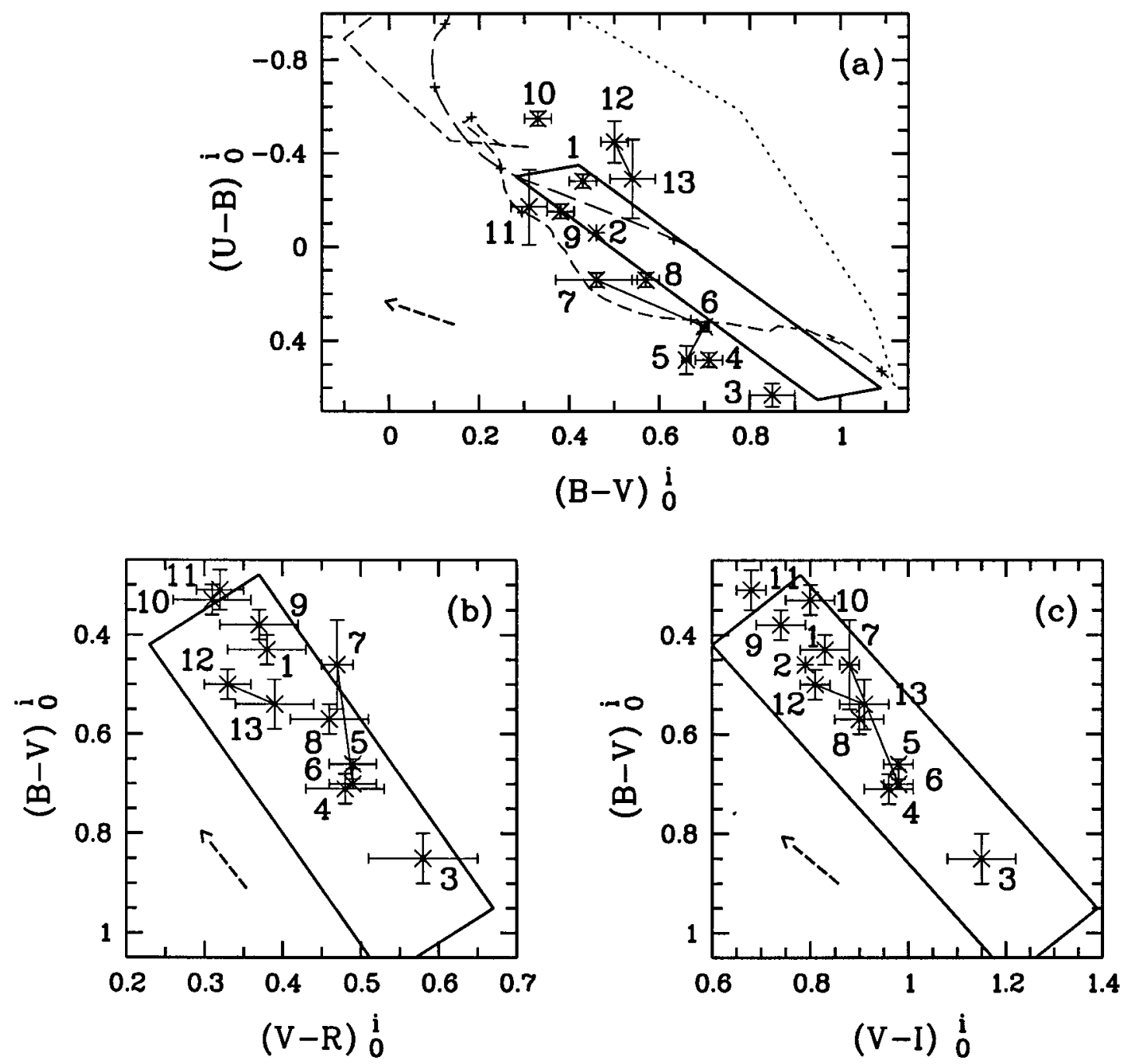

Fig. 8. Two-color diagrams: a) $(U-B)_{0}^{i}$ vs. $(B-V)_{0}^{i}$, b) $(B-V)_{0}^{i}$ vs. $(V-R)_{0}^{i}$, and c) $(B-V)_{0}^{i}$ vs. $(V-I)_{0}^{i}$ for different regions in NGC 2336 . The boxes enclose the observed positions of the integrated color indices for galaxies which follow the normal color sequence (NCS), as calculated in Buta \& Williams (1995). The dashed curves in a) represent evolutionary tracks for stellar systems with: constant star formation rate (long dashed curve), and an instantaneous starburst (short dashed curve), with the objects ageing (and reddening) from $1 \mathrm{Myr}$ to 15 Gyr during their approach to the NCS (the small vertical crosses indicate ages of: $10 \mathrm{Myr}, 100 \mathrm{Myr}, 1 \mathrm{Gyr}$ and $10 \mathrm{Gyr}$ ). These tracks were calculated using the PEGASE2 code (see Fioc \& Rocca-Volmerange 1997). The dotted curve towards the top right of a) shows the color change resulting from a starburst in an old stellar system (age $15 \mathrm{Gyr}$ ), from Fioc \& Rocca-Volmerange (1997), with the young (age 1 Myr) population mass fraction changing from $10^{-6} M_{\text {total }}$ (bottom) to $10^{-3} M_{\text {total }}$ (top). The short dashed arrows in the bottom left of each plot show the direction of the correction for internal absorption due to dust in the galaxy. The vertical and horizontal bars show the color index measurement errors for each region of the galaxy. The numbers denoting these regions are explained in the text (see Sect. 3.4).

within $28 \mathrm{kpc}\left(180^{\prime \prime}\right)$ of the center; 2 is the total color index of the galaxy, from the LEDA Database; 3 is the integrated color of the nucleus, within $0.3 \mathrm{kpc}\left(2^{\prime \prime}\right)$ of the center; 4 is the integrated color of the bulge, at distances of $0.6-2.5 \mathrm{kpc}\left(4^{\prime \prime}-16^{\prime \prime}\right)$ from the center; 5, 6 and 7 are the color indices of the bar, at distances of: 13"' 3,18 '. 1 and 22'. 5 from the center, respectively (corresponding to deprojected distances of: $3.2 \mathrm{kpc}, 4.4 \mathrm{kpc}$ and $5.5 \mathrm{kpc}$, respectively); 8 is the integrated color of the ring, at distances of $5.0-5.6 \mathrm{kpc}\left(32^{\prime \prime}-36^{\prime \prime}\right)$ from the center; 9 is the integrated color of the inner part of the disk, at distances of $10.6-16.3 \mathrm{kpc}\left(68^{\prime \prime}-104^{\prime \prime}\right)$ from the center; 10 is the integrated color of the outer part of the disk, at distances of $16.3-25.9 \mathrm{kpc}$ $\left(104^{\prime \prime}-166^{\prime \prime}\right)$ from the center; 11 is the color index of the spiral arms; and 12,13 are the colors of the interarm regions, for the western and eastern parts of the disk, respectively.

Most of the points in the two-color diagrams lie along the normal color sequence (NCS) for galaxies (Figs. 8a-c); this is supporting evidence for a uniform history of star formation in NGC 2336 (or, in general, for any history with local $S F E^{1}=$ constant $)$. There appears to be a small displacement of the points in the $(U-B)_{0}^{i}$ vs. $(B-V)_{0}^{i}$ two-color diagram; this may be the result of additional internal dust absorption in NGC 2336. The color indices of the galaxy as a whole are typical of Sbc type spiral galaxies (points 1, 2 in Figs. 8a-c).

${ }^{1} S F E \equiv\left(\mathrm{d} M_{\mathrm{s}} / \mathrm{d} t\right) M_{\text {gas }}^{-1}$, where $\mathrm{d} M_{\mathrm{s}} / \mathrm{d} t$ is the mass of stars formed in the time interval $\mathrm{d} t$, and $M_{\mathrm{gas}}$ is the gas mass. 
There is no current star formation activity, either in the nucleus, or in the bulge, of the galaxy (the locations of points 3 and 4 are typical for old stellar systems without recent star formation activity).

The bar of NGC 2336 becomes bluer towards the edges (points 5-7 in Figs. 8a-c); so the luminosity contribution from young stars increases towards the ends of the bar. The offset of these points to the left of the NCS in the $(U-B)_{0}^{i}$ vs. $(B-$ $V)_{0}^{i}$ two-color diagram may be the result of a complex stellar population content (i.e. a mixture of both young and old stars) in the bar (Gusev 2000).

In general, the fraction of young stellar populations increases with distance from the galaxy center. The most active star formation regions occur in the outer parts of the disk and spiral arms (see points $10-13$ in Figs. 8a-c). Nevertheless, the star formation history in the disk appears to differ from that in the spiral arms; the colors of the spiral arms (point 11) are characteristic of a stellar system with constant SFE (Figs. 8b, c), whereas the colors of the outer disk of NGC 2336 (point 10) are typical for a stellar system with a relatively small contribution from a young stellar population (Figs. 8a, c).

The color difference between the western and eastern parts of the disk of NGC 2336 (points 12 and 13, respectively) can be explained by the reddening expected from the internal dust absorption due to the inclination of the galaxy (see Figs. 8a-c and Table 3), as well as by the concentration of dust in the inner borders of the spiral arms.

\subsection{Star formation regions in the galaxy}

We studied the properties of the 34 largest star formation regions in NGC 2336 (including 5 in the southern star formation complex). The results of our investigations are presented in Figs. 9, 10a-c, 11a-c and in Table 5; listed in this table are the positions, color indices, diameters, ages, intrinsic reddenings and distances to the galaxy center, which we determined for each of these star formation regions.

In contrast to the previous subsections, and Figs. 8a-c, the "true" color indices of the star formation regions were considered in this subsection; these "true" intensities were obtained by subtracting the background intensity of the spiral arms from the integrated intensities in the areas occupied by the star formation regions.

The diameter of each star formation region was determined from the FWHM for regions having a starlike profile, or else by the distance between the points of maximal light gradient for regions having extended profiles. The diameter for a non-spherical region was determined along its minor axis.

The ages and intrinsic reddenings of the star formation regions were estimated from their positions in the two-color diagrams, using evolutionary tracks of synthetically aged stellar systems generated by the PEGASE2 code (Fioc \& Rocca-Volmerange 1997). Kennicutt's (1983) initial mass function (IMF) and a simple stellar population (SSP) model were used. A graphical description of the method used to estimate the ages and reddenings is shown in Fig. 9 for a typical star formation region, plotted in the $(U-B)_{0}^{i}$ vs. $(B-V)_{0}^{i}$ twocolor diagram.

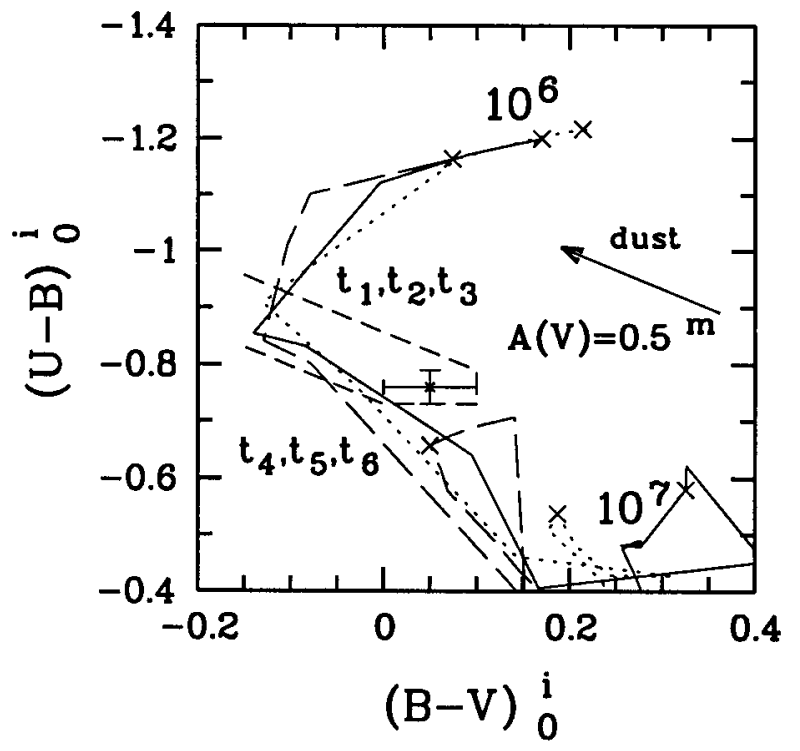

Fig. 9. Graphical description of the method used to estimate the ages and intrinsic reddenings for star formation regions, by their positions in the two-color diagram. Evolutionary tracks of synthetically aged stellar systems with $Z=0.008$ (dotted curve), 0.02 (solid curve), and 0.05 (long dashed curve), are shown. The numbers $\left(10^{6}\right.$ and $\left.10^{7}\right)$ are the ages of the synthetic systems in years. The arrow gives the direction of the correction for dust absorption, and its length corresponds to an extinction of $A(V)=0.5$ mag. Color index error bars are shown for a typical star formation region.

The area surrounded by the short dashed lines in Fig. 9 shows possible real positions for the star formation region indicated in the $(U-B)_{0}^{i}$ vs. $(B-V)_{0}^{i}$ two-color diagram. The uncertainties are the result of observational errors and unknown intrinsic reddening. This intrinsic reddening is due to the dust absorption in star formation regions; it is an additional local absorption to the general Galactic absorption, and to the absorption due to the inclination of NGC 2336.

The symbols $t_{1}-t_{6}$ in Fig. 9 denote the intersection points of the model evolutionary tracks with the perimeter of the area enclosing the possible real positions of the indicated star formation region. So, $t_{1}, t_{2}$ and $t_{3}$ are the minimum possible ages of this region, for the models with $Z=0.05,0.008$ and 0.02 , respectively; and $t_{4}, t_{5}$ and $t_{6}$ are the corresponding maximum possible ages.

In our estimation method, we proceeded in two steps. Firstly, we estimated a minimum $\left(t_{\min }^{U B V}\right)$ and a maximum $\left(t_{\max }^{U B V}\right)$ possible age for the star formation region, using the $(U-B)_{0}^{i}$ vs. $(B-V)_{0}^{i}$ two-color diagram:

$$
\begin{aligned}
& t_{\min }^{U B V}=\min \left(t_{1}, t_{2}, t_{3}\right) \\
& \min \left(t_{1}, t_{2}, t_{3}\right) \equiv \min \left[t_{Z=0.05}, t_{Z=0.008}, t_{Z=0.02}\right], \\
& t_{\max }^{U B V}=\max \left(t_{4}, t_{5}, t_{6}\right) \\
& \max \left(t_{4}, t_{5}, t_{6}\right) \equiv \max \left[t_{Z=0.05}, t_{Z=0.008}, t_{Z=0.02}\right]
\end{aligned}
$$

The estimation of a minimum $\left(A_{\min }^{U B V}(V)\right)$ and a maximum $\left(A_{\max }^{U B V}(V)\right)$ possible intrinsic absorption was carried out in an analogous way. For the chosen (typical) star formation region, we obtained the following results: $t_{\min }^{U B V}=3.4 \mathrm{Myr}, t_{\max }^{U B V}=$ $5.6 \mathrm{Myr}, A_{\min }^{U B V}(V)=0 \mathrm{mag}, A_{\max }^{U B V}(V)=0.66 \mathrm{mag}$. 
This first step is also used for independently estimating these parameters from the $(B-V)_{0}^{i}$ vs. $(V-R)_{0}^{i}$ and $(B-V)_{0}^{i}$ vs. $(V-I)_{0}^{i}$ two-color diagrams.

The second step yields the final estimated parameters from combining the results derived from each of the three two-color diagrams:

$$
\begin{aligned}
& t_{\min }=\max \left(t_{\min }^{U B V}, t_{\min }^{B V R}, t_{\min }^{B V I}\right), \\
& t_{\max }=\min \left(t_{\max }^{U B V}, t_{\max }^{B V R}, t_{\max }^{B V I}\right) .
\end{aligned}
$$

$A_{\min }(V)=\max \left[A_{\min }^{U B V}(V), A_{\min }^{B V R}(V), A_{\min }^{B V I}(V)\right]$,

$A_{\max }(V)=\min \left[A_{\max }^{U B V}(V), A_{\max }^{B V R}(V), A_{\max }^{B V I}(V)\right]$.

A more detailed description of this method has been presented in Gusev (2002).

The estimated parameters of the star formation regions are presented in Table 5. Successive columns of this table present the following properties for each of the 34 regions: (1) assigned sequence number, (2) apparent coordinates, in arcseconds relative to the galaxy center, (3-6) $(U-B)_{0}^{i},(B-V)_{0}^{i},(V-R)_{0}^{i}$ and $(V-I)_{0}^{i}$ color indices, (7) characteristic diameter $D$ in pc, (8) estimated age $T$ in Myr, (9) estimated value of the intrinsic reddening (or color excess) $E(B-V)^{2}$ due to dust absorption in the star formation region, and (10) deprojected distance $r$ from the center of NGC 2336 to the star formation region, in kpc.

The ages $T$ and the intrinsic reddening values $E(B-V)$ of the star formation regions, as estimated by their positions in the two-color diagrams, are consistent with each other, with the exception of seven regions $(10,11,17,18,21,27 d$, and 28), whose ages were estimated with relatively large errors.

Most of the NGC 2336 star formation regions are located within compact areas in the two-color diagrams (Figs. 10a-c), with the exception of several very young regions (e.g. 14, 15, 23), and those with large intrinsic reddening (e.g. 20, 27).

The positions of six star formation regions $(5,9,13,14,18$ and 29$)$ in the $(B-V)_{0}^{i}$ vs. $(V-R)_{0}^{i}$ diagram do not lie on any of the synthetic evolutionary tracks (see Fig. 10b). However, we estimated their ages and intrinsic reddening values from their positions in the other two-color diagrams (i.e. $(U-B)_{0}^{i}$ vs. $(B-V)_{0}^{i}$ and $(B-V)_{0}^{i}$ vs. $\left.(V-I)_{0}^{i}\right)$; we obtained mutually consistent results for five of these six regions. Four have typical parameters for star formation regions in NGC 2336, with the exception of the $(V-R)_{0}^{i}$ color index (see 5, 9, 13 and 29 in Table 5); we are unable to explain the positions of these four outliers in the $(B-V)_{0}^{i}$ vs. $(V-R)_{0}^{i}$ diagram, in terms of the PEGASE2 stellar evolutionary models that we used. The parameters of the sixth region (18) were estimated using only the $(B-V)_{0}^{i}$ vs. $(V-I)_{0}^{i}$ diagram.

The positions of the NGC 2336 star formation regions in the two-color diagrams are displaced from the evolutionary tracks for synthetically aged systems (see Figs. 10a, c); this is due to the varying amounts of dust absorption "intrinsic" to each of the regions. As estimated from the two-color diagrams, this absorption is in the range $A_{V}=0.3-1.5 \mathrm{mag}$ for the star formation regions in NGC 2336.

\footnotetext{
${ }^{2}$ Ratio value $A_{V} / E(B-V)=3.10$ was used.
}
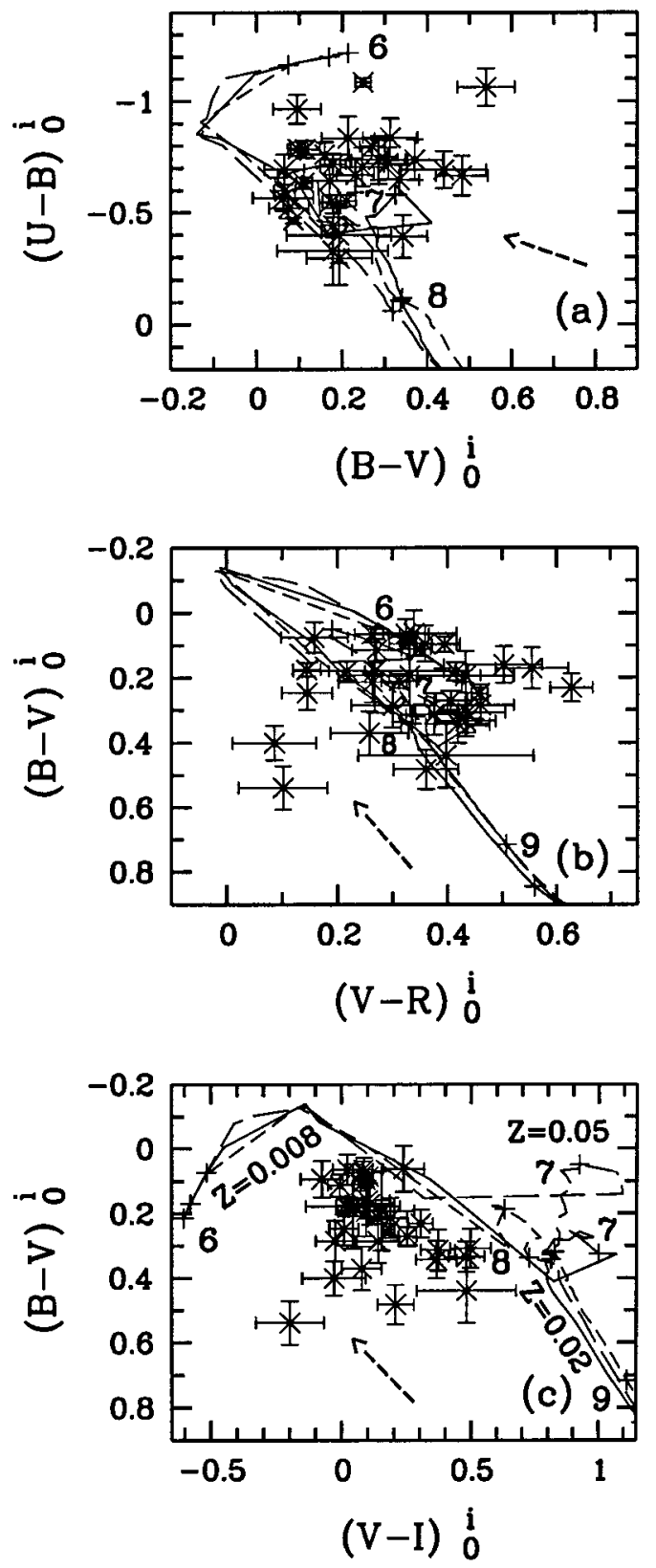

Fig. 10. Two-color diagrams: a) $(U-B)_{0}^{i}$ vs. $(B-V)_{0}^{i}$, b) $(B-V)_{0}^{i}$ vs. $(V-R)_{0}^{i}$, and c) $(B-V)_{0}^{i}$ vs. $(V-I)_{0}^{i}$, for the NGC 2336 star formation regions. Evolutionary tracks of synthetically aged stellar systems, with $Z=0.008$ (short dashed curves), 0.02 (solid curves), and 0.05 (long dashed curves), are shown. Small vertical crosses indicate system ages of: 1, 10, $100 \mathrm{Myr}$ and $1 \mathrm{Gyr}$. The numbers are the base 10 logarithms of the system ages in years. The color index error bars are shown. Short dashed arrows give the direction of the correction for internal dust absorption in the galaxy.

Most of the star formation regions identified in this paper have diameters in the range $250-500$ pc (Fig. 11a). Since the atmospheric seeing during our observations was $2^{\prime \prime}$ (corresponding to a linear scale of $\sim 300 \mathrm{pc}$ ), we cannot detect star formation regions smaller than this limit. Five out of the six regions with diameters $D>500 \mathrm{pc}$ are complex (the exception is region 28 with $D=565 \mathrm{pc}$ ). Apart from this particular case, there 
Table 5. Parameters of star formation regions in NGC 2336 (available in electronic form).

\begin{tabular}{llllllllll}
\hline \hline$N$ & $X^{\prime \prime}, Y^{\prime \prime}$ & $(U-B)_{0}^{i}$ & $(B-V)_{0}^{i}$ & $(V-R)_{0}^{i}$ & $(V-I)_{0}^{i}$ & $D, \mathrm{pc}$ & $T, \mathrm{Myr}$ & $E(B-V)$ & $r, \mathrm{kpc}$ \\
\hline
\end{tabular}

is no simple (i.e. non-complex) star formation region with diameter $D>500$ pc in NGC 2336.

Both observational selection effects and the chosen age estimation method influence the age histogram we derived for the star formation regions in NGC 2336 (Fig. 11b). The ages for these regions are our best estimates, given the observed data and evolutionary tracks available to us. In general, evolutionary models for synthetic stellar systems consistently predict a maximum in emitted $B$ luminosity in the age range $2-4 \mathrm{Myr}$, irrespective of the mass of the system. Nevertheless, the age histogram shows no evidence for any recent star formation burst in the galaxy (i.e. within the past $6 \mathrm{Myr}$ ); recent star formation in NGC 2336 appears to have proceeded continuously, rather than intermittently.

Three out of five star formation regions older than 5.5 Myr are located in the southern star formation complex of NGC 2336; it seems that active, recent, star formation in this complex began about $6 \mathrm{Myr}$ ago.

A concentration of star formation regions towards the ring, in the range $12-16 \mathrm{kpc}$ radius, was found (Fig. 11c). The outer part of the southern star formation complex is located at the same distance from the galaxy center. The radius of this star formation ring coincides with that of the molecular $\left(\mathrm{H}_{2}\right)$ ring (at $13.6 \mathrm{kpc}$, see Sect. 1) in the galaxy. This clearly indicates that these star formation regions are concentrated in the molecular ring of NGC 2336.

\section{Discussion}

NGC 2336 is a typical intermediate type spiral galaxy, having properties of both early and late type galaxies. The large bulge of this galaxy, with $L_{\text {bulge }}(I) / L_{\text {disk }}(I)=0.16$, is typical of early type galaxies. On the other hand, the short, exponentiallike light profile of the bar is more typical of late type galaxies (Elmegreen \& Elmegreen 1985).

It is likely that exponential-like bars in late type galaxies extend out only to the inner Lindblad resonance (Elmegreen \& Elmegreen 1985). We estimated a corotation resonance radius for NGC 2336, using the method given in Canzian (1998). The galaxy has an approximately flat rotation curve (Biviano et al. 1991; Keel 1996). If $R_{\text {in }}$ and $R_{\text {out }}$ are the observed values of the innermost and outermost radii of the spiral structure, respectively, then following Canzian (1998), the corotation resonance radius $R_{\mathrm{CR}}$ can be limited to the range:

$(2-\sqrt{2}) R_{\text {out }} \leq R_{\mathrm{CR}} \leq(2+\sqrt{2}) R_{\text {in }}$.

For NGC 2336, we estimated $R_{\text {in }}=5.4 \mathrm{kpc}$, and $R_{\text {out }}=$ $24.1 \mathrm{kpc}$, thus yielding $R_{\mathrm{CR}}=16 \pm 2 \mathrm{kpc}$ from Eq. (7). This value is approximately equal to both the radius of the star formation ring, and that of the molecular ring. We suspect that the most active star formation takes place at the corotation resonance radius.
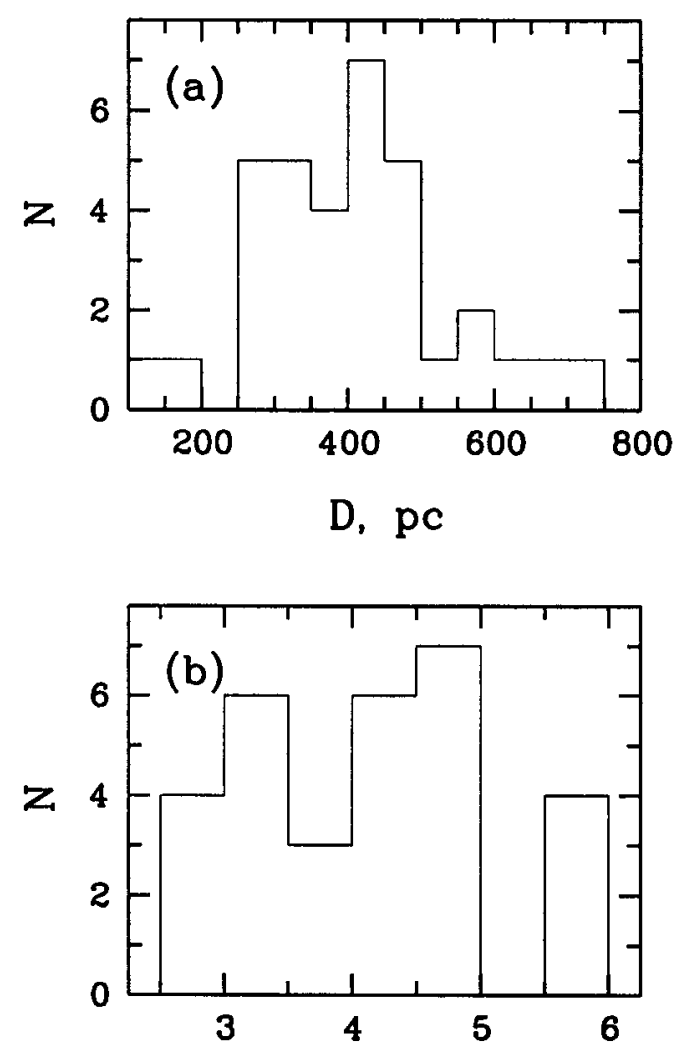

T, Myr

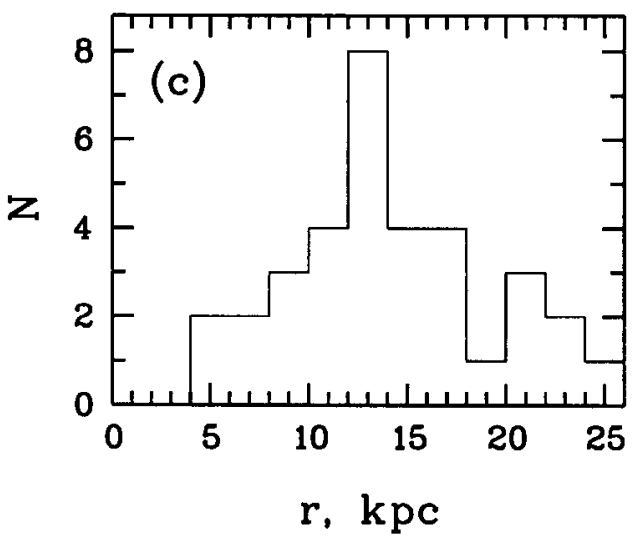

Fig. 11. Number distribution histograms of the star formation regions in NGC 2336, by: a) diameter, b) age, and c) deprojected distance from the galaxy center.

Boroson (1981), Grosbol (1985), and Baggett et al. (1998) measured a disk scale length for NGC 2336 in the optical, but they obtained contradictory results: a scale length of $r_{0}=55^{\prime \prime}$ was found by Grosbol (1985) and Baggett et al. (1998), versus $70^{\prime \prime}$ by Boroson (1981). This is likely to be due to the different radial ranges used in these studies. In their statistical investigations of large samples of spiral galaxies, Grosbol (1985) 
and Baggett et al. (1998) included the inner disk regions in their determinations of the disk scale lengths; they measured the disks from the galaxy center out to a radial angular distance of $r=20^{\prime \prime}$. However, this approach is incorrect, as chance distributions of the brightest spiral arms and star formation regions distort the radial light distribution function (see Fig. 7a). In addition, the slope of the radial light distribution (and the value of the disk scale length) depends on the observational passbands used for the inner parts of the disk of NGC 2336.

Our results show that NGC 2336 is a galaxy with active star formation in the disk, with a star formation rate typical of late type spirals. The recent star formation in this galaxy has proceeded continuously, not intermittently (i.e. without distinct star formation bursts). The colors of the outer disk are typical of an old stellar system with a small fractional contribution from a young stellar population, as judged from the two-color diagrams. We suspect that this results from a mixture of the relatively old stellar population in the outer disk, with the young population residing in numerous star formation regions.

\section{Conclusions}

From the multicolor surface photometry analysis of NGC 2336, we conclude that:

1. An exponential-like disk, with a scale length of $11.0 \pm$ $0.1 \mathrm{kpc}\left(71^{\prime \prime} \pm 1^{\prime \prime}\right)$ and a large bulge with an effective radius of $1.8 \pm 0.2 \mathrm{kpc}\left(12^{\prime \prime} \pm 1^{\prime \prime}\right)$ have been observed in NGC 2336. The bulge has a de Vaucouleurs radial light distribution profile. The bulge-disk luminosity ratio is $L_{\text {bulge }} / L_{\text {disk }}=0.16 \pm 0.04$ in the I passband.

2. Corrected for inclination, the semi-length of the bar in NGC 2336 is 5.9 kpc (37'.5). The bar has an exponential-like intensity profile. The scale length of the eastern part of the bar is $\alpha^{-1}=2.8 \pm 0.3 \mathrm{kpc}\left(18^{\prime \prime} \pm 2^{\prime \prime}\right)$ in the $I$ passband.

3. NGC 2336 is a galaxy with active star formation ongoing in the disk. There is no current star formation activity either in nucleus or in the bulge. Recent star formation in NGC 2336 has proceeded continuously, not intermittently (i.e. without distinct star formation bursts).

4. The color difference between the western and eastern parts of the disk in NGC 2336 can be explained by the internal dust absorption due to the inclination of the galaxy, as well as by the concentration of the dust to the inner borders of the spiral arms.

5. The star formation regions are concentrated towards the molecular ring in NGC 2336. With the sole exception of one region, there is no simple (i.e. non-complex) star formation region larger than $500 \mathrm{pc}$ in diameter.

6. Active, recent, star formation in the southern complex of NGC 2336 began about 6 Myr ago.

Acknowledgements. ASG was supported by a grant of the Postdoctoral Program at Kyungpook National University (20002001), and by grants No. 01-02-16800 and No. 01-02-17597 from the Russian Foundation for Basic Research. MGP was supported by grant No. R01-1999-00023 from the Korea Science and Engineering
Foundation (KOSEF). The authors are grateful to B.-C. Lee (Bohyunsan Optical Astronomy Observatory) for assistance with the observations, and to A. B. Fletcher (Korea Astronomy Observatory) for help with editing this paper. ASG would like to thank A. V. Zasov (Sternberg Astronomical Institute) for helpful discussions. The authors thank the anonymous referee for valuable remarks.

\section{References}

Aaronson, M., Huchra, J., Mould, J. R., et al. 1982, ApJS, 50, 241

Baggett, W. E., Baggett, S. M., \& Anderson, K. S. J. 1998, AJ, 116, 1626

Bartunov, O. S., Tsvetkov, D. Yu., \& Filimonova, I. V. 1994, PASP, 106, 1276

Biviano, A., Girardi, M., Giuricin, G., Mardirossian, F., \& Mezzetti, M. 1991, ApJ, 376, 458

Blackman, C. P., \& van Moorsel, G. A. 1984, MNRAS, 208, 91

Boroson, T. 1981, ApJS, 46, 177

Buta, R., \& de Vaucouleurs, G. 1983, ApJS, 51, 149

Buta, R., \& Williams, K. L. 1995, AJ, 109, 543

Canzian, B. 1998, ApJ, 502, 582

de Vaucouleurs, G., de Vaucouleurs, A., Corwin, J. R., et al. 1991, Third Reference Catalogue of Bright Galaxies (New York: Springer)

Elmegreen, B. G., \& Elmegreen, D. M. 1985, ApJ, 288, 438

Fioc, M., \& Rocca-Volmerange, B. 1997, A\&A, 326, 950

Grosbol, P. J. 1985, A\&AS, 60, 261

Gusev, A. S. 2000, AZh, 77, 654

Gusev, A. S. 2002, IAU Symp., 207, 450

Heckman, T. M. 1980, A\&A, 87, 152

Heraudeau, Ph., \& Simien, F. 1996, A\&AS, 118, 111

Hodge, P. W., \& Kennicutt, R. C. Jr. 1983, AJ, 88, 296

Hummel, E., Pedlar, A., Davies, R. D., \& van der Hulst, J. M. 1985, A\&AS, 60, 293

Keel, W. C. 1993, AJ, 106, 1771

Keel, W. C. 1996, ApJS, 106, 27

Kennicutt, R. C., Jr. 1983, ApJ, 272, 54

Landolt, A. U. 1992, AJ, 104, 340

Martin, P. 1995, AJ, 109, 2428

Martin, M. C. 1998, A\&AS, 131, 77

Niklas, S., Klein, U., Braine, J., \& Wielebinski, R. 1995, A\&AS, 114, 21

Paturel, G., Andernach, H., Bottinelli, L., et al. 1997, A\&AS, 124, 109

Prugniel, P., \& Heraudeau, Ph. 1998, A\&AS, 128, 299

Quillen, A. C., McDonald, C., \& Alonso-Herrero, A., et al. 2001, ApJ, 547,129

Rownd, B. K., \& Young, J. S. 1999, AJ, 118, 670

Schechter, P. L. 1983, ApJS, 52, 425

Schneider, S. E., \& Salpeter, E. E. 1992, ApJ, 385, 32

Tifft, W. G. 1982, ApJS, 50, 319

van den Bergh, S. 1995, AJ, 110, 613

van Driel, W., de Graauw, T., de Jong, T., \& Wesselius, P. R. 1993, A\&AS, 101, 207

van Moorsel, G. A. 1983, A\&AS, 54, 19

van Moorsel, G. A. 1987, A\&A, 176, 13

Wilke, K., Mollenhoff, C., \& Matthias, M. 1999, A\&A, 344, 787

Wunderlich, E., \& Klein, U. 1991, A\&AS, 87, 247

Xu, C., Lisenfeld, U., Volk, H. J., \& Wunderlich, E. 1994, A\&A, 282, 19

Young, J. S., Xie, S., Tacconi, L., et al. 1995, ApJS, 98, 219

Young, J. S., Allen, L., Kenney, J. D. P., Lesser, A., \& Rownd, B. 1996, AJ, 112, 1903 
A. S. Gusev and M.-G. Park: Structure and stellar population of NGC 2336, Online Material p 1

\section{Online Material}


Table 2. Journal of observations of NGC 2336.

\begin{tabular}{|c|c|c|c|c|}
\hline Filter & $\begin{array}{c}\text { Exposure } \\
\text { time }\end{array}$ & $\begin{array}{c}\text { Start UT } \\
\text { 2001 Feb. } 22\end{array}$ & Seeing & Airmass \\
\hline$i$ & $120 \mathrm{~s}$ & $10^{\mathrm{h}} 28^{\mathrm{m}}$ & 1..9 & 1.43 \\
\hline$i$ & $180 \mathrm{~s}$ & $10^{\mathrm{h}} 36^{\mathrm{m}}$ & $1 . .7$ & 1.43 \\
\hline$r$ & $180 \mathrm{~s}$ & $10^{\mathrm{h}} 42^{\mathrm{m}}$ & 1..7 & 1.42 \\
\hline$r$ & $240 \mathrm{~s}$ & $10^{\mathrm{h}} 48^{\mathrm{m}}$ & 1". 8 & 1.42 \\
\hline$v$ & $240 \mathrm{~s}$ & $10^{\mathrm{h}} 56^{\mathrm{m}}$ & $2 . .1$ & 1.42 \\
\hline$v$ & $240 \mathrm{~s}$ & $11^{\mathrm{h}} 03^{\mathrm{m}}$ & 2.0 & 1.41 \\
\hline$b$ & $300 \mathrm{~s}$ & $11^{\mathrm{h}} 10^{\mathrm{m}}$ & 1..9 & 1.41 \\
\hline$b$ & $300 \mathrm{~s}$ & $11^{\mathrm{h}} 18^{\mathrm{m}}$ & $2 . .0$ & 1.41 \\
\hline$u$ & $300 \mathrm{~s}$ & $11^{\mathrm{h}} 26^{\mathrm{m}}$ & $2 . .3$ & 1.40 \\
\hline$u$ & $300 \mathrm{~s}$ & $11^{\mathrm{h}} 35^{\mathrm{m}}$ & $2 . .2$ & 1.40 \\
\hline$u$ & $300 \mathrm{~s}$ & $11^{\mathrm{h}} 43^{\mathrm{m}}$ & $2 . .3$ & 1.40 \\
\hline
\end{tabular}

Table 5. Parameters of star formation regions in NGC 2336.

\begin{tabular}{|c|c|c|c|c|c|c|c|c|c|}
\hline$N$ & $X^{\prime \prime}, Y^{\prime \prime}$ & $(U-B)_{0}^{i}$ & $(B-V)_{0}^{i}$ & $(V-R)_{0}^{i}$ & $(V-I)_{0}^{i}$ & $D, \mathrm{pc}$ & $T, \mathrm{Myr}$ & $E(B-V)$ & $r, \mathrm{kpc}$ \\
\hline 1 & $148.6 N, 31.3 W$ & $-0.74 \pm 0.11$ & $0.29 \pm 0.07$ & $0.30 \pm 0.08$ & $0.14 \pm 0.08$ & 190 & $3.0 \pm 1.0$ & $0.37 \pm 0.09$ & 24.1 \\
\hline 2 & $142.0 N, 11.3 E$ & $-0.78 \pm 0.04$ & $0.11 \pm 0.03$ & $0.27 \pm 0.04$ & $-0.01 \pm 0.05$ & 435 & $3.4 \pm 0.5$ & $0.18 \pm 0.07$ & 22.8 \\
\hline 3 & $128.8 N, 12.4 E$ & $-0.83 \pm 0.10$ & $0.21 \pm 0.06$ & $0.26 \pm 0.06$ & $0.17 \pm 0.07$ & 515 & $3.5 \pm 0.5$ & $0.27 \pm 0.12$ & 20.8 \\
\hline 4 & $111.9 N, 31.0 E$ & $-0.74 \pm 0.09$ & $0.29 \pm 0.06$ & $0.46 \pm 0.06$ & $-0.02 \pm 0.07$ & 290 & $2.5 \pm 1.1$ & $0.30 \pm 0.08$ & 20.2 \\
\hline 5 & $95.6 \mathrm{~N}, 13.3 \mathrm{~W}$ & $-0.67 \pm 0.08$ & $0.23 \pm 0.04$ & $0.63 \pm 0.04$ & $0.30 \pm 0.05$ & 345 & $4.7 \pm 1.2$ & $0.25 \pm 0.14$ & 15.1 \\
\hline 6 & $88.6 N, 4.5 W$ & $-0.74 \pm 0.09$ & $0.37 \pm 0.07$ & $0.26 \pm 0.07$ & $0.08 \pm 0.08$ & 580 & $3.0 \pm 0.7$ & $0.40 \pm 0.11$ & 13.9 \\
\hline 7 & $79.6 N, 4.8 E$ & $-0.52 \pm 0.05$ & $0.08 \pm 0.05$ & $0.16 \pm 0.06$ & $0.09 \pm 0.06$ & 640 & $5.5 \pm 0.1$ & $<0.11$ & 12.7 \\
\hline 8 & $68.0 N, 34.6 E$ & $-0.80 \pm 0.04$ & $0.27 \pm 0.03$ & $0.41 \pm 0.03$ & $0.25 \pm 0.04$ & 135 & $3.3 \pm 0.8$ & $0.35 \pm 0.07$ & 14.9 \\
\hline 9 & $62.3 N, 48.4 W$ & $-0.70 \pm 0.09$ & $0.25 \pm 0.05$ & $0.15 \pm 0.05$ & $0.01 \pm 0.06$ & 475 & $4.2 \pm 1.4$ & $0.31 \pm 0.13$ & 15.6 \\
\hline 10 & $52.4 N, 0.2 W$ & $-0.39 \pm 0.10$ & $0.34 \pm 0.06$ & $0.42 \pm 0.06$ & $0.37 \pm 0.08$ & 485 & $<45 ?$ & $<0.52$ & 8.2 \\
\hline 11 & $48.9 N, 9.7 E$ & $-0.29 \pm 0.12$ & $0.19 \pm 0.08$ & $0.43 \pm 0.09$ & $0.09 \pm 0.11$ & 295 & $<9$ & $<0.38$ & 8.3 \\
\hline 12 & $37.6 \mathrm{~N}, 78.6 \mathrm{~W}$ & $-0.69 \pm 0.07$ & $0.06 \pm 0.05$ & $0.32 \pm 0.04$ & $0.02 \pm 0.06$ & 450 & $4.5 \pm 0.8$ & $0.15 \pm 0.09$ & 21.5 \\
\hline 13 & $36.5 N, 42.3 E$ & $-0.76 \pm 0.06$ & $0.16 \pm 0.06$ & $0.50 \pm 0.07$ & $0.10 \pm 0.08$ & 375 & $4.1 \pm 1.1$ & $0.24 \pm 0.09$ & 13.3 \\
\hline 14 & $34.0 \mathrm{~N}, 32.8 \mathrm{~W}$ & $-1.06 \pm 0.08$ & $0.54 \pm 0.07$ & $0.10 \pm 0.08$ & $-0.20 \pm 0.13$ & 355 & $<2.4$ & $0.33 \pm 0.07$ & 9.9 \\
\hline 15 & $24.8 N, 62.0 E$ & $-1.08 \pm 0.02$ & $0.25 \pm 0.02$ & $0.46 \pm 0.01$ & $0.18 \pm 0.02$ & 360 & $<3.0$ & $0.09 \pm 0.08$ & 17.6 \\
\hline 16 & $18.4 \mathrm{~N}, 38.5 \mathrm{~W}$ & $-0.84 \pm 0.09$ & $0.31 \pm 0.06$ & $0.38 \pm 0.05$ & $0.37 \pm 0.07$ & 315 & $3.5 \pm 0.3$ & $0.36 \pm 0.13$ & 10.6 \\
\hline 17 & $17.6 \mathrm{~N}, 85.2 \mathrm{~W}$ & $-0.63 \pm 0.03$ & $0.11 \pm 0.02$ & $0.35 \pm 0.02$ & $0.07 \pm 0.03$ & 460 & $<6.2$ & $<0.20$ & 23.0 \\
\hline 18 & $15.2 \mathrm{~N}, 27.7 \mathrm{~W}$ & - & $0.40 \pm 0.05$ & $0.09 \pm 0.08$ & $-0.03 \pm 0.12$ & 295 & $<3.5 ?$ & $0.31 \pm 0.13$ & 7.7 \\
\hline 19 & $3.3 N, 43.7 W$ & $-0.60 \pm 0.03$ & $0.07 \pm 0.02$ & $0.26 \pm 0.03$ & $0.08 \pm 0.04$ & 405 & $4.6 \pm 1.1$ & $<0.22$ & 11.8 \\
\hline 20 & $8.5 S, 21.6 E$ & $-0.69 \pm 0.08$ & $0.44 \pm 0.10$ & $0.40 \pm 0.16$ & $0.48 \pm 0.19$ & 485 & $3.0 \pm 1.2$ & $0.51 \pm 0.16$ & 5.9 \\
\hline 21 & $26.4 S, 48.3 W$ & $-0.46 \pm 0.01$ & $0.09 \pm 0.01$ & $0.33 \pm 0.01$ & $0.10 \pm 0.02$ & 430 & $<5.8$ & $<0.23$ & 14.1 \\
\hline 22 & $27.9 S, 9.8 E$ & $-0.65 \pm 0.07$ & $0.33 \pm 0.05$ & $0.43 \pm 0.05$ & $0.48 \pm 0.07$ & 260 & $4.5 \pm 0.9$ & $0.44 \pm 0.08$ & 4.9 \\
\hline 23 & $37.7 \mathrm{~S}, 60.4 \mathrm{~W}$ & $-0.96 \pm 0.06$ & $0.09 \pm 0.06$ & $0.36 \pm 0.06$ & $-0.08 \pm 0.08$ & 305 & $2.6 \pm 0.7$ & $0.14 \pm 0.05$ & 17.9 \\
\hline 24 & $37.8 S, 48.7 E$ & $-0.79 \pm 0.03$ & $0.10 \pm 0.02$ & $0.39 \pm 0.02$ & $0.09 \pm 0.03$ & 420 & $4.0 \pm 0.5$ & $0.10 \pm 0.05$ & 13.9 \\
\hline 25 & $41.2 S, 33.7 E$ & $-0.55 \pm 0.03$ & $0.18 \pm 0.02$ & $0.14 \pm 0.03$ & $0.01 \pm 0.03$ & 650 & $4.6 \pm 2.1$ & $<0.34$ & 10.7 \\
\hline 26 & $46.8 S, 9.8 E$ & $-0.72 \pm 0.07$ & $0.31 \pm 0.06$ & $0.44 \pm 0.07$ & $0.50 \pm 0.08$ & 415 & $4.0 \pm 0.3$ & $0.40 \pm 0.10$ & 7.6 \\
\hline $27 \mathrm{a}$ & $72.4 S, 0.2 E$ & $-0.57 \pm 0.06$ & $0.06 \pm 0.07$ & $0.34 \pm 0.08$ & $0.24 \pm 0.08$ & 325 & $5.6 \pm 0.4$ & $<0.16$ & 11.4 \\
\hline $27 b$ & $77.0 S, 3.6 E$ & $-0.40 \pm 0.12$ & $0.19 \pm 0.12$ & $0.33 \pm 0.13$ & $0.15 \pm 0.13$ & 720 & $5.5 \pm 0.4$ & $<0.34$ & 12.0 \\
\hline $27 \mathrm{c}$ & $79.9 S, 6.9 E$ & $-0.67 \pm 0.09$ & $0.48 \pm 0.06$ & $0.36 \pm 0.06$ & $0.21 \pm 0.07$ & 280 & $2.8 \pm 1.0$ & $0.52 \pm 0.08$ & 12.5 \\
\hline $27 d$ & $82.1 S, 9.1 E$ & $-0.55 \pm 0.03$ & $0.21 \pm 0.02$ & $0.31 \pm 0.03$ & $0.14 \pm 0.04$ & 320 & $4.1 \pm 2.7$ & $<0.35$ & 12.9 \\
\hline $27 \mathrm{e}$ & $83.3 S, 4.0 E$ & $-0.33 \pm 0.15$ & $0.18 \pm 0.13$ & $0.27 \pm 0.15$ & $0.04 \pm 0.18$ & 425 & $5.5 \pm 0.3$ & $<0.31$ & 13.0 \\
\hline 28 & $89.2 S, 39.9 E$ & $-0.44 \pm 0.05$ & $0.18 \pm 0.03$ & $0.42 \pm 0.02$ & $0.06 \pm 0.03$ & 565 & $4.5 \pm 2.7$ & $<0.32$ & 16.9 \\
\hline 29 & $94.3 S, 37.2 E$ & $-0.64 \pm 0.10$ & $0.17 \pm 0.06$ & $0.55 \pm 0.07$ & $0.11 \pm 0.09$ & 400 & $4.4 \pm 1.0$ & $0.25 \pm 0.12$ & 17.1 \\
\hline 30 & $108.9 S, 31.6 E$ & $-0.72 \pm 0.05$ & $0.18 \pm 0.03$ & $0.22 \pm 0.03$ & $0.02 \pm 0.04$ & 455 & $3.5 \pm 0.3$ & $0.29 \pm 0.03$ & 18.4 \\
\hline
\end{tabular}

